\title{
Mechanochemistry in polymers with supramolecular mechanophores
}

\author{
Alexander P. Haehnel, ${ }^{1, \#}$ Yoshimitsu Sagara, ${ }^{1, \#}$ Yoan C. Simon, ${ }^{1 *}$ and Christoph Weder ${ }^{1 *}$ \\ ${ }^{1}$ Adolphe Merkle Institute, University of Fribourg, Chemin des Verdiers 4, 1700 Fribourg, Switzerland \\ ${ }^{\#}$ These authors contributed equally. \\ * To whom correspondence should be addressed: yoan.simon@unifr.ch, christoph.weder@unifr.ch
}

\section{Introduction}

Touch is one of the most primal senses in living organisms and can be broadly defined as the ability to transduce a mechanical cue into an electrical signal, which in turn can be transcribed by the cerebrum.[1] Whether dealing with stretching motions, hair movement or pressure, the detection of mechanical stimuli is based on mechano-transducing cells that are capable of generating events that eventually lead to the creation of an action potential that travels along the afferent neuron. The paradigm for cellular transduction is based on the opening of transmembrane proteins, which allow the formation of an ionic imbalance that triggers the electrical signaling. It is important to recognize the general non-covalent nature of the mechanotransduction pathways in living cells. In an attempt to emulate such pathways and bestow mechanoresponsiveness upon synthetic materials systems, researchers have begun to utilize weak bonds to impart polymers with new, mechanically triggered functionalities such as catalysis, healability, and color or $\mathrm{pH}$ changes.[2-4] While many of the recently developed materials rely on mechanically responsive motifs - also referred to as mechanophores that dissociate upon cleavage of weak covalent bonds, a subset of mechanically responsive materials has emerged that is based on the dissociation of assemblies formed through weak, dynamic, noncovalent interactions. The development of such materials systems roots in the realization that a myriad of natural processes happen through weak interactions, $[5,6]$ such as the opening of transmembrane proteins, pre-catalytic enzyme-substrate complexes, or actin polymerization. While some of the early work in the area of polymer mechanochemistry had largely focused fundamental experiments that often involved sonochemical processes in dilute solution,[7-11] there has been a growing interest in solid materials that are capable of selectively transducing macroscopic mechanical forces into molecular events that trigger desirable macroscopic materials responses.[3, 9, 12, 13] Indeed, this emerging design approach has recently permitted the development a range of adaptive solid-state polymers in which pre-programmed functions can be triggered by external stimuli, including mechanical triggers.[14] While the general aspects of mechanochemistry with polymers have been the subject of several recent reviews,[15-18] this report summarizes the development of polymer mechanochemistry involving non-covalent mechanophores at the hand of pertinent examples from the recent literature. The materials are organized according to the types of noncovalent interactions exploited (pi-pi, metal-ligand, hydrogen-bonding). This account encompasses an extensive number of examples of molecular level experiments - mostly based on sonochemistry in solutions - and also emphasizes mechanochemical effects in solid-state materials. Since there are many examples of thixotropic gels or otherwise mechanically responsive physical gels, the present article does not cover extensively this burgeoning field of supramolecular chemistry. However, a selection of key systems that have influenced the direction of the field is presented in relevant contexts.

\section{2. $\pi-\pi$ Interactions}

Whether transduction is based on the scission or disassembly of covalent or non-covalent bonds, one of the perhaps most useful and most widely investigated mechanically triggered responses is a color change. The latter is an effective reporting handle that rapidly indicates the occurrence of a predefined mechanical transduction event. Consequently, mechanochromic materials have garnered a great deal of attention in the past decade, as they provide a one-of-a-kind solution to harness mechanical force and investigate stress transfers down to the molecular level.[19] In organic materials, colors and color changes are often introduced by utilizing (poly)aromatic molecules with unique optical properties. In such systems, $\pi-\pi$ interactions have been shown play a critical role in the 
mechanochemical transduction process, since the absorption and/or photoluminescence properties of aggregated organic or organometallic molecules are often significantly different from those of individual molecules.[20, 21] Some chromophores undergo complete quenching of the photoluminescence in their aggregated states, others exhibit aggregation-induced emission, while a third groups displays different photoluminescence and/or absorption colors in assembled or isolated (or monomeric) states. Different molecular origins lead so such responses, including excimer formation,[22, 23] charge-transfer complexes[24, 25] or exciton coupling,[26] and changes in molecular mobility.[27] Thus, it is possible to influence the photophysical properties of polymer-based materials by altering the aggregation state of embedded $\pi$-conjugated chromophores through the application of mechanical forces.[28-31]

One of the first attempts to strategically use the mechanically induced disassembly of aggregated chromophores in a polymer matrix involved a family of fluorescent dyes which form (static) excimers in their aggregated states.[32] Such complexes between two identical photoluminescent (PL) molecules, one in an electronically excited and one in its ground state, were discovered by Förster and Kasper sixty years ago.[33, 34] The excimer has a lower potential energy than the separated components (i.e. a molecule in its first electronically excited state and a molecule in its ground state) and therefore excimers emit at higher wavelengths (lower energy) than the monomer species from which they are constituted (in line with the common terminology, the term 'monomer' is used here to describe optical processes that involve single-molecule excited states, as opposed to excimers). In solution, excimer formation is frequently a dynamic, diffusion-controlled process. [35, 36] By contrast, diffusion of dye molecules in solid polymers is slow in comparison to the lifetime of the excited states, so that in this environment excimers predominantly arise from preformed ground-state aggregates, which may also display different absorption characteristics than the monomeric species.[37]

Early work on the mechanically induced deformation of supramolecular assemblies of chromophores in polymer matrices was inspired by the surprising discovery that solid-state tensile deformation of blends of minute amounts of the highly fluorescent, conjugated polymer poly(2,5-(2ethylhexyloxy)-p-phenylene ethynylene) (EHO-OPPE) and ultra-high-molecular-weight poly(ethylene) not only led to uniaxial orientation of the conjugated guest molecules along the elongational axis, but also promoted the break-up of small aggregates comprising a few of these molecules into well-individualized structures (Figure 1).[38] This process was monitored by singlemolecule spectroscopy (Figure 2) and did not cause any noticeable color changes. While the study did also not involve well-defined supramolecular assemblies, the results paved the way for subsequent work on mechanochromic polymers comprising assemblies of aggregachromic, excimer-forming cyano-substituted oligo( $p$-phenylene vinylene)s (cyano-OPVs, Figures 2-4). [39-54]

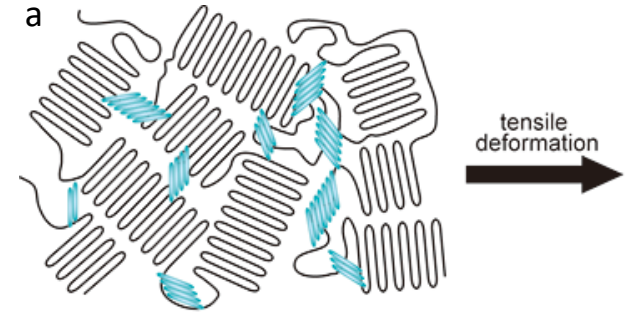

: polymer

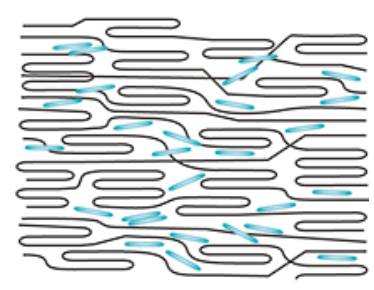

: : dye

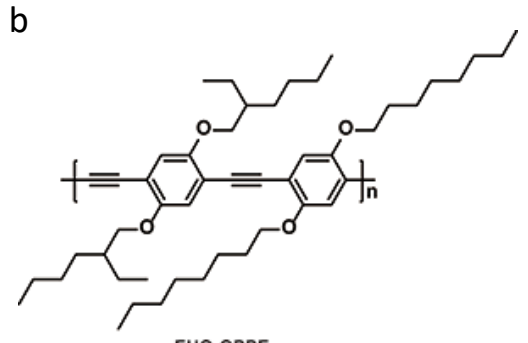

EHO-OPPE

Figure 1. a) Schematic representation of the mechanically induced transformation of a disordered semicrystalline polymer comprising small aggregates of self-assembled chromophores (small-molecule or polymeric) into an oriented structure in which the guest molecules are well dispersed. b) Structure of EHO-OPPE, a poly(p-phenylene ethynylene) derivative. 


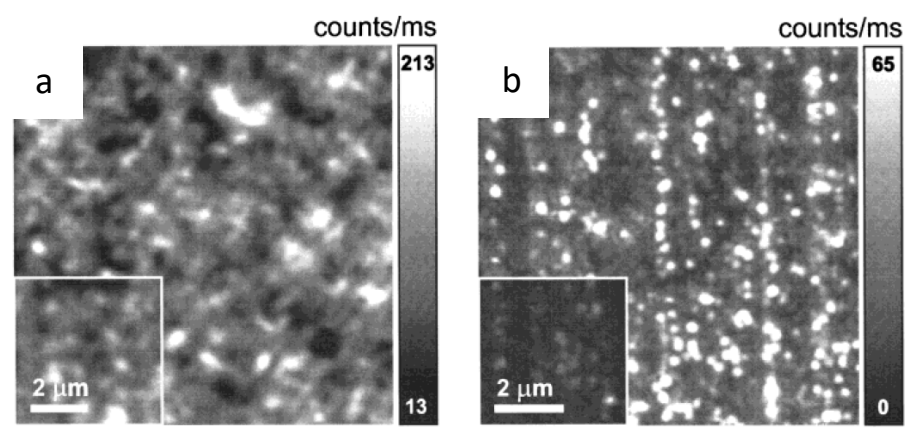

Figure 2. Scanning confocal microscopy images of blend films of ultra-high-molecular-weight polyethylene and $10^{-4} \% \mathrm{w} / \mathrm{w}$ EHO-OPPE (Figure 1). a) As prepared film. b) Uniaxially deformed sample drawn to a draw ratio of 80. Both images were acquired by detecting the polarization direction oriented parallel to the (eventual) deformation direction. The inset show images acquired by detecting the orthogonal polarization direction. Adapted with permission from ref.[38]. Copyright 2000 American Chemical Society.

Building on the above-summarized findings, Löwe and Weder reported the first example of mechanoresponsive luminescent polymers comprising self-assembled cyano-OPVs in 2002.[55] This chromophore family was chosen due to several attractive features, including high thermal stability, ease of color tuning through peripheral group functionalization (Figure 3a), and a strong tendency towards excimer formation. The significant red-shift of the emission maxima (up to $140 \mathrm{~nm}$ ) upon aggregation makes them especially interesting, since the photoluminescence color changes can be easily detected by the naked eye (Figure 3b-c). Furthermore, cyano-OPVs are readily accessible and their solubility and aggregation behavior in different host polymers can easily be modified via the choice of the peripheral groups. The electronic properties of cyano-OPVs can be tuned over a wide range and depend on both, the electron donating or accepting character of substituents of attached to the conjugated core, [56, 57] as well as the nature of the supramolecular assemblies.[44, 58] For example, in C1-RG (red-to-green), the introduction of two alkoxy groups into the central aromatic ring reduces the HOMO-LUMO gap compared to the C1-YB (yellow-to-blue) analog, resulting in a shift of the emission color in solution as well as in the crystalline state (Figure 3). The crystal structure of C1-RG features a parallel arrangement of neighboring molecules, which organize so that the electron-poor cyano-vinylene moiety of one molecule overlaps with the electron-rich central ring of its neighbor. These assemblies display excimer emission. Surprisingly, increasing the length of the peripheral alkyloxy groups in the YB series to dodecyl (C12-YB) or octadecyloxy (C18-YB) leads to crystal structures in which the molecules do not adopt a "staggered" array and where blue monomer emission is dominant.[Error! Bookmark not defined.] This observation shows that $\pi-\pi$ and aliphatic (or other) interactions in cyano-OPVs must be carefully balanced in order to achieve self-assembled structures that support excimer formation.

a)

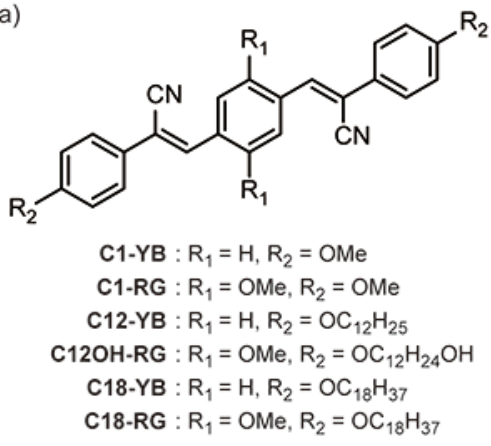

b)

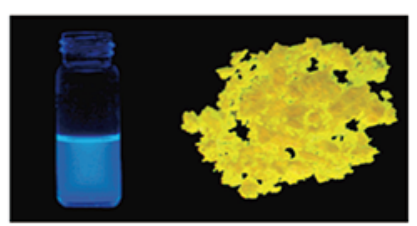

c)

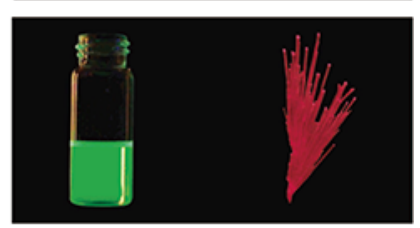

Figure 3. a) Examples of molecular structures of cyano-OPVs. b, c) Images of C1-YB (b) and C1-RG (c) recorded under excitation with ultraviolet light in chloroform (left) and in the crystalline state (right). Panel $\mathrm{b}$ and $\mathrm{c}$ reprinted with permission from ref. [39]. Copyright 2003 American Chemical Society.

A guest-diffusion technique was employed to introduce C1-YB and C1-RG into linear low-density poly(ethylene) (LLDPE). Through optimization of the dyeing conditions, i.e., dye concentration, temperature and swelling time, cyano-OPV-doped polymer films that exhibit a mechanically induced 
photoluminescence color change were produced. Figure 4 shows the emission spectra of a C1-RGdoped LLDPE film at different draw ratios. In the undrawn state, samples with sufficiently high dye content display a broad emission band with maximum at $650 \mathrm{~nm}$, characteristic of excimer emission. Emission bands with well-resolved vibronic structures at $470-570 \mathrm{~nm}$ are also observed, which correspond to emission from molecularly dispersed chromophores. Figure 4 shows clearly that with increasing strain, the intensity of the excimer band decreases relative to the monomer emission bands. This relative intensity change suggests that nano-scale aggregates of C1-RG, which are present in the as-prepared polymer film, are converted into monomeric species in response to tensile deformation, as depicted in Figure 1a. Thus, even though the functionality-imparting motifs are not covalently attached to the polymer matrix, stresses can effectively be transferred and cause the disassembly of aggregates that are presumably small and held together by weak, non-covalent forces. In the context of currently applied terminology, the cyano-OPV stacks should probably be referred to as the mechanophores, although it can be argued that it would be appropriate to utilize the term for the cyano-OPV motif itself. It is noted that this comment applies to many of the mechanically responsive moieties discussed in this text.

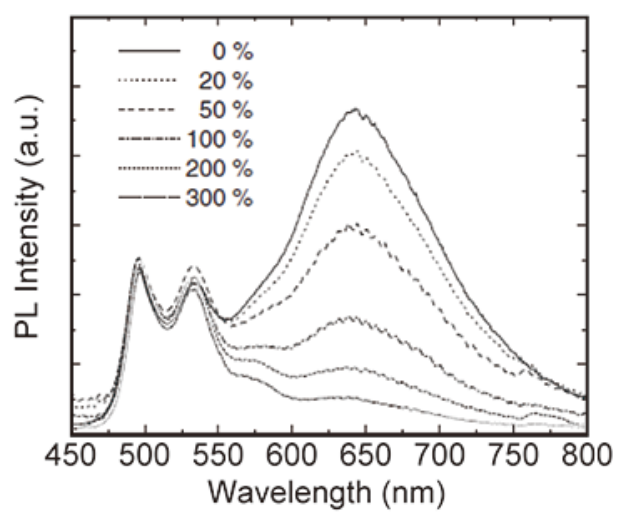

Figure 4. Photoluminescence spectra of an LLDPE/C1-RG blend as function of draw ratio. Adapted with permission from ref.[55]. Copyright 2002 Wiley-VCH.

Crenshaw et al. subsequently demonstrated that mechanically responsive blends can also be produced by melt-processing LLDPE/cyano-OPV blends and presented a detailed investigation on the phase-behavior of these materials.[39] To avoid large-scale phase separation, blend films were prepared by melt-mixing and compression molding thin films at $180{ }^{\circ} \mathrm{C}$ (choosing the dye concentration so that at this temperature thermodynamic miscibility is achieved; in case of C1-RG, only $0.18 \% \mathrm{w} / \mathrm{w}$ were used) and subsequent quenching the blends into a kinetically-trapped state. Due to the sub-ambient glass transition temperature of LLDPE, the blends are thermodynamically unstable and the dyes slowly aggregate under ambient conditions. The process was shown to span several months by monitoring the increase of the excimer emission band (Figure 5a). The aggregation process was significantly accelerated when the blend films were immersed in hexane (Figure 5b), which swells the LLDPE and increases the translational mobility of the guest molecules, but is a poor solvent for C1-RG. Mechanically responsive behavior was demonstrated for polymer blends in which the cyanoOPVs had been allowed to aggregate by either of the above-mentioned processes. The photoluminescence color of blend films comprising either C1-RG or C1-YB exhibited a significant change upon tensile deformation (Figure 6a), concomitant with a substantial reduction of the relative intensity of the excimer bands (Figure $6 \mathrm{~b}$ and $\mathrm{c}$ ). When LLDPE/C1-RG blends were slowly cooled from the melt, the formation of large dye aggregates with dimensions exceeding several $\mu \mathrm{m}$ was observed, which could not be mechanically dispersed, leading to materials in which no mechanochromic response was observed. 
a)

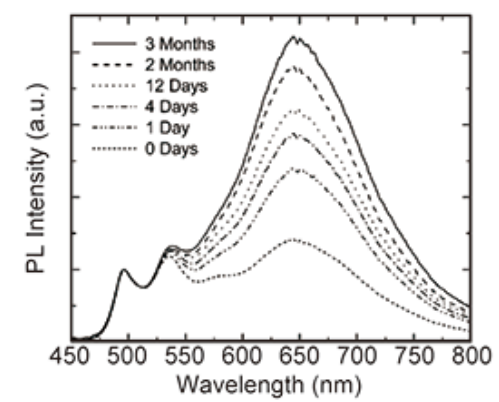

b)

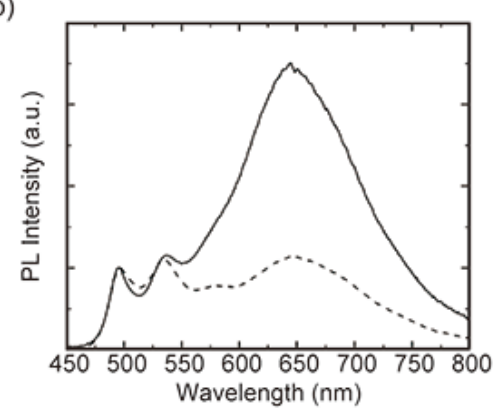

Figure 5. a) Photoluminescence spectra of a blend film of LLDPE and $0.18 \% \mathrm{w} / \mathrm{w}$ C1-RG as function of storage time under ambient conditions. b) Photoluminescence spectra of a blend film of LLDPE and $0.18 \% \mathrm{w} / \mathrm{w}$ C1-RG freshly prepared (dashed line) and after swelling the film for $15 \mathrm{~min}$ in hexane and subsequent drying (solid line). All spectra were normalized to the intensity of the monomer peak. Adapted with permission from ref.[39]. Copyright 2003 American Chemical Society.

a)

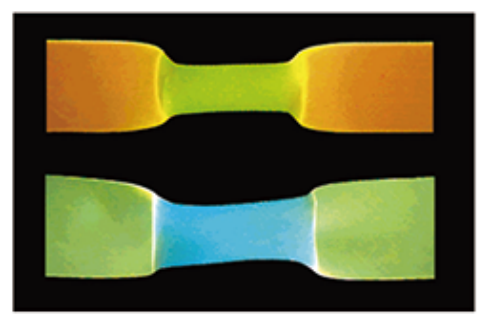

b)

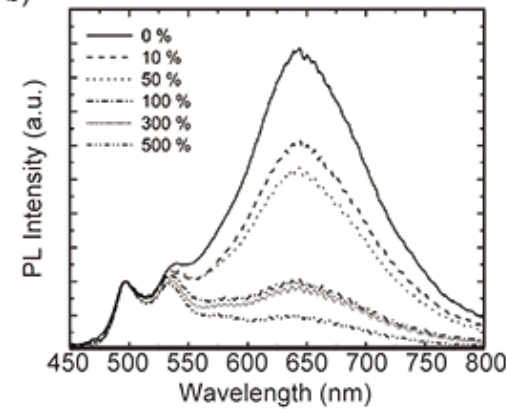

c)

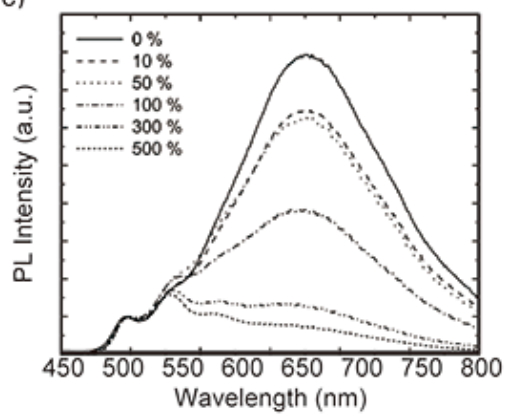

Figure 6. a) Pictures of blend films of LLDPE and 0.18\% w/w C1-GR (top) and 0.20\% w/w C1-YB (bottom) stretched at room temperature. The pictures were taken under excitation with ultraviolet light. b, c) Photoluminescence spectra of blend films of LLDPE and $0.20 \% \mathrm{w} / \mathrm{w}$ C1-GR (b) or C1-YB (c) as a function of draw ratio. All spectra were normalized to the intensity of the monomer peak. Adapted with permission from ref. [39]. Copyright 2003 American Chemical Society.

Based on the above-discussed discovery that the size of excimer-forming aggregates matters, Crenshaw et al. subsequently investigated how substituents attached to the lateral benzene rings of cyano-OPVs influence the aggregation behavior of these dyes in poly(ethylene) blends.[40] C18-RG, featuring octadecyloxy instead of methoxy groups in the lateral positions (Figure 3a), was shown to nucleate much more efficiently than C1-RG. The comparably inefficient nucleation of C1-RG appears to limit the number of dye aggregates formed, which results in the slow growth of larger aggregates. Smaller dye aggregates of C18-RG in LLDPE film are more easily dispersed during tensile deformation, leading to a more substantial photoluminescence color change compared to their C1-RG analogs. However, it appears that also in the case of C18-RG a semicrystalline matrix is required or at least beneficial to nucleate dye crystal growth. Other effects on the mechanoresponsive luminescence properties, i.e., the ability of the polymer host to transfer stress and break up the chromophore aggregates upon deformation have also been investigated, such as matrix crystallinity or polarity. In poly(ethylene) with higher crystallinity, dye aggregates are more easily disassembled than in poly(ethylene) with lower crystallinity. The ability of the polymer host to break up dye aggregates upon deformation, and thus the extent of color change observed upon deformation, is related to the plastic deformation process of PE crystallites - specifically those arranged in a lamellar morphology and increases with increasing polymer crystallinity, decreasing dye aggregate size, and decreasing rates of deformation. The color-strain traces (where color was expressed by the ratio of monomer to excimer emission intensity, $\mathrm{I}_{\mathrm{M}} / \mathrm{I}_{\mathrm{E}}$ ) of PE/C18-RG blends blends all mirror the stress-strain profiles. In these cases $\mathrm{I}_{\mathrm{M}} / \mathrm{I}_{\mathrm{E}}$ increases steeply upon yielding, grows moderately during neck propagation, and displays a slightly steeper increase during strain hardening. Beyond poly(ethylene)s, the Weder group has used poly(ethylene terephthalate) [41] and a semicrystalline fluoropolymer [42] as host matrices to create mechanochromic materials based on cyano-OPVs.

Reversible, mechanically induced dissociation of cyano-OPVs has been achieved in thermoplastic elastomers in which the chromophores were covalently incorporated into the polymer backbones.[43] Although chromophore aggregation and disassembly in these materials are intermolecular processes, 
the design is reminiscent of more recently developed mechanoresponsive polymers with built-in "mechanophores", i.e., weak links that are designed to break upon application of a load to the polymer chain. Several elastomers were prepared and the hard segment to soft segment ratio and the dye content were systematically varied. Upon deformation, all compression-molded, quenched polymer films containing C12OH-RG showed a combination of monomer and excimer emission. In the best compositions, the prepared polyurethane exhibited a pronounced change of the photoluminescence color from an orange-red, excimer-dominated emission to green fluorescence that is characteristic of molecularly dispersed chromophores (Figure 7a and b). In situ opto-mechanical studies, in which samples were exposed to a cyclic triangular strain pattern, demonstrated that the fluorescence color change nicely mirrored the reversible stress-strain response of the polymer (Figure 7c).

a)

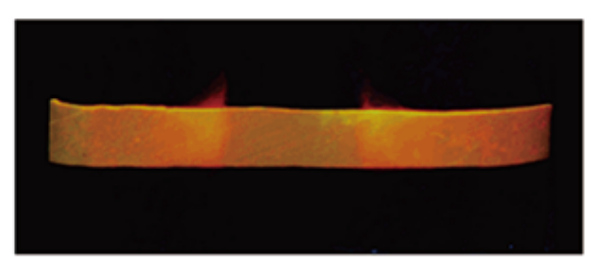

b)

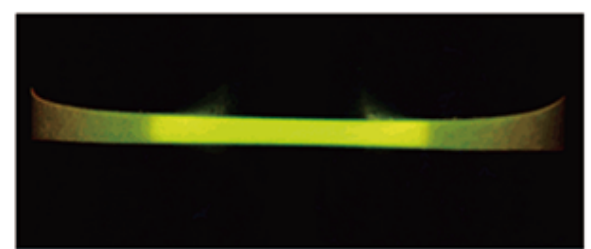

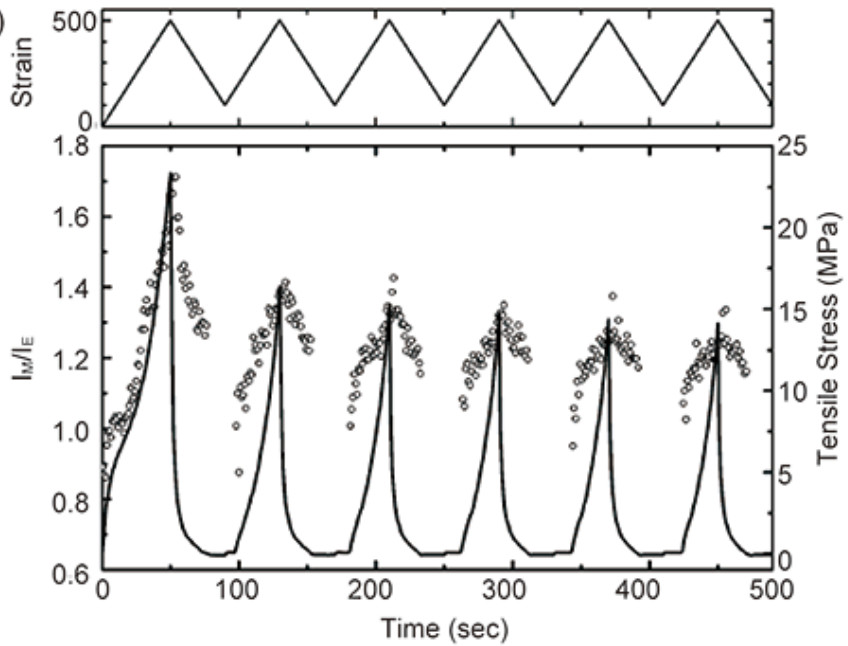

Figure 7. a) Picture of a mechanochromic elastomer made by integrating $\mathbf{C 1 2 O H}-\mathbf{R G}$ into a thermoplastic polyurethane backbone in the unstretched state. b) Picture of the same material in the stretched state. Both pictures were taken under illumination with ultraviolet light. c) Ratio of monomer to excimer emission $\mathrm{I}_{\mathrm{M}} / \mathrm{I}_{\mathrm{E}}$ (circles) and tensile stress (solid line) under a triangular strain cycle between $0 \%$ and $500 \%$ at frequency of $0.0125 \mathrm{~Hz}$. Adapted with permission from ref. [43]. Copyright 2006 American Chemical Society.

While the property of interest in all of the above-reported cyano-OPV containing materials was a mechanically induced change of the photoluminescence color (i.e., a mechanically induced transformation from an excimer-rich to a monomer-dominated emission), certain cyano-OPVs also display a changed of their absorption color upon (dis)assembly. Thus, Kunzelman et al. reported mechanochromic blend films based on C18-RG and either poly(ethylene terephthalate glycol) (PETG)[45] or LLDPE.[44] Figure 8 shows how the absorption spectrum (Figure 8a) and visual appearance (Figure 8b) of an LLDPE/C18-RG blend film change in response to solid-state tensile deformation. The spectral shift indicates that small dye aggregates are molecularly dispersed upon elongation. Similar changes in absorption have also been reported for a PETG-based polymer films in which a cyano-OPV dye was covalently introduced into polymer chain.[45]

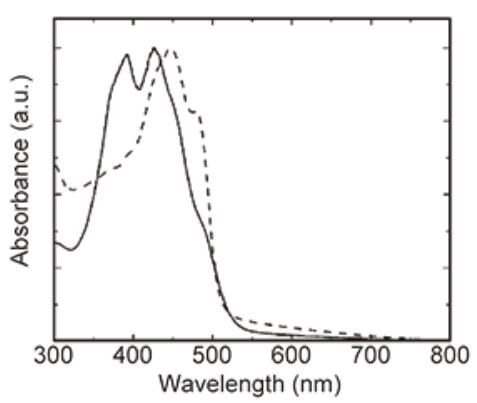

b)

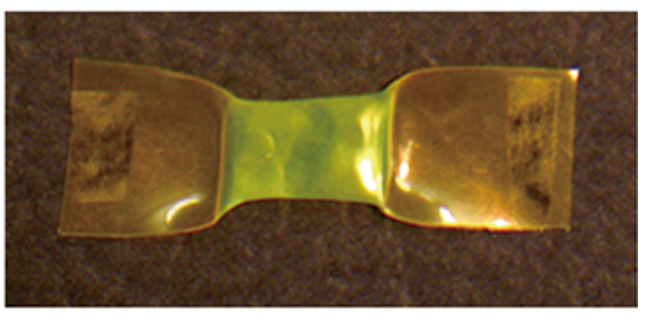

Figure 8. a) Normalized absorption spectra of a $0.5 \% \mathrm{w} / \mathrm{w}$ LLDPE/C18-RG blend film before (solid line) and after (dashed line) stretching at room temperature to a draw ratio of ca. $300 \%$. b) Picture of the same material in which the center portion 
has been uniaxially deformed. The image was taken under ambient illumination. Adapted with permission from ref.[44]. Copyright 2006 Wiley-VCH.

Beyond cyano-OPVs, several other dyes that form excimers in their aggregated state have also been used for mechanosensing purposes. Pucci and coworkers have reported that poly(propylene) (PP) films containing bis(benzoxazolyl)stilbene (BBS) (Figure 9a) change the photoluminescence color upon tensile deformation. [52] In the as-prepared state, the emission spectrum (Figure 9b) shows a broad excimer emission band around $500 \mathrm{~nm}$, along with sharper monomer emission bands at 410, 430 and $455 \mathrm{~nm}$. Mechanical deformation lowers the intensity of the excimer band, resulting in a photoluminescence color change (Figure 9c). Mechanosensing behavior of blend films of poly(1,4butylene succinate) and BBS have also investigated by the same group.[53]

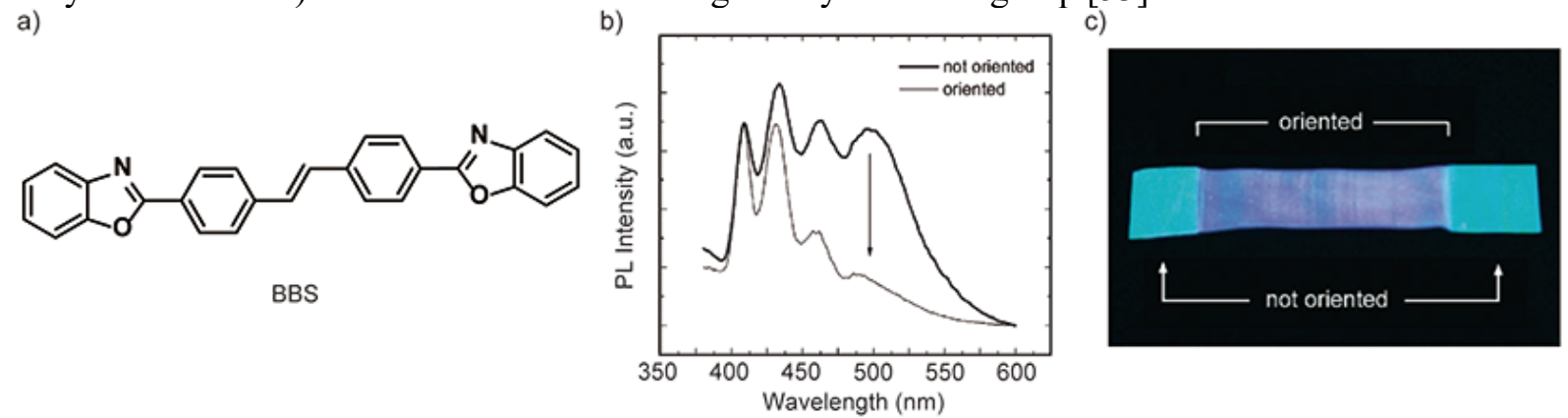

Figure 9. a) Molecular structure of bis(benzoxazolyl)stilbene (BBS). b) Fluorescence spectra of a PP/BBS blend film containing $0.5 \% \mathrm{w} / \mathrm{w}$ of BBS before and after tensile deformation c) Picture of the same material in which the center portion was uniaxially deformed. The image was taken under excitation with ultraviolet light. Panel $\mathrm{b}$ and $\mathrm{c}$ adapted with permission from ref.[52]. Copyright 2005 Wiley-VCH.

Pucci and coworkers have also reported LLDPE-based films containing perylene bisimide derivatives,[54] which have been intensively examined because of their attractive optoelectronic character and propensity to assemble into supramolecular architectures. Blend films comprising $N, N^{\prime}-$ bis-(2'-ethylhexyl)perylene-3,4,9,10-tetracarboxyldiimide (EE-PTCDI) in LLDPE were obtained by melt-mixing procedures and blend films with $0.1 \% \mathrm{w} / \mathrm{w}$ of the dye exhibited mechanoresponsive behavior as shown in Figure 10. The intensity of the emission band around $620 \mathrm{~nm}$, which is ascribed to emission from EE-PTCDI aggregates, decreased drastically after drawing, indicating that the perylene compound became molecularly dispersed. Also, the transition dipole moment of EE-PTCDI was shown to align along the direction of drawing.

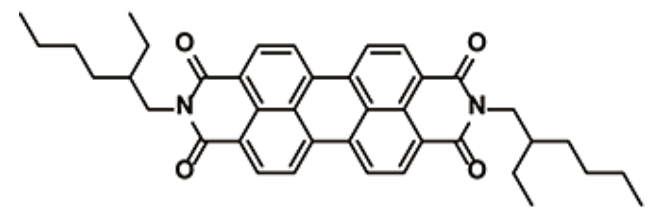

EE-PTDCI

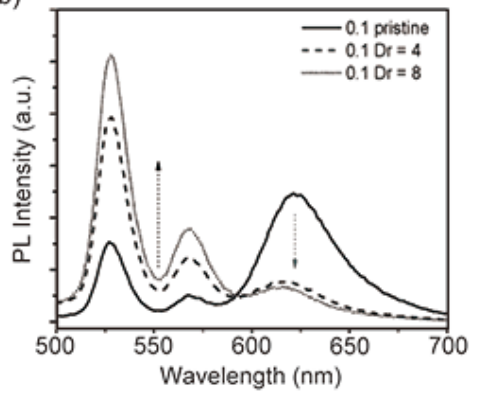

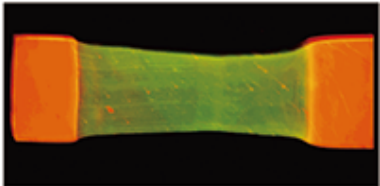

Figure 10. a) Molecular structure of $N, N^{\prime}$-bis-(2'-ethylhexyl)perylene-3,4,9,10-tetracarboxyldiimide (EE-PTCDI). b) Fluorescence spectra of a LLDPE/EE-PTDCI blend film containing $0.1 \% \mathrm{w} / \mathrm{w}$ EE-PTDCI before and after uniaxial deformation to different draw ratios (Dr). c) Picture of the same material in which the center portion has been uniaxially deformed to a draw-ratio of 4 . The image was taken under excitation with ultraviolet light. Panel $\mathrm{b}$ and $\mathrm{c}$ adapted with permission from ref. [54]. Copyright 2008 American Chemical Society.

Most of the materials systems described above are based on blends of conventional polymers to which small amounts of self-assembling polymers were added. However, the covalent attachment of mechanoresponsive luminescent molecular assemblies on the surface of polymers. Sagara et al. have 
recently reported poly(lactic acid) (PLA) beads, that feature mechanoresponsive luminescent micelles on their surface (Figure 11).[59] The latter consist of 1,6-disubstituted pyrene derivative Py1 (Figure 12), and were attached to the surface of the PLA beads through covalent bonds. Compound Py1 selfassembles in aqueous conditions to form micelles, on account of its amphiphilic nature. Upon application of a sufficiently large mechanical forces to the micelles (in aqueous conditions), their photoluminescence color changes from yellow to green. The yellow emission is ascribed to excimer formation of the luminescent core of Py1. Using the amino groups introduced in the peripheral positions of the dendrons, the resulting micelles could be covalently attached to a variety of surfaces that had also been decorated with amine groups using the amine-reactive crosslinker L2 (Figure 12). Thus, the authors succeeded in attaching the micelles to PLA beads $(\Phi=70-150 \mu \mathrm{m})$ with amino groups on their surfaces, which was confirmed by confocal fluorescence microscopy. Mechanical stimulation was applied by vortexing the glass vial containing water and the micelle-decorated PLA beads. A photoluminescent color change could easily be observed by naked eye when the PLA beads having the mechanoresponsive micelles were vortexed in the presence of glass beads, which are heavier than PLA beads. By contrast, no obvious change in emission spectra was observed when PLA beads with having the micelles were vortexed in the absence of said glass beads. The mechanoresponsive luminescent PLA beads seem to have a threshold below which no photoluminescence color change occurs. This threshold behavior is consistent with a uniform aggregate size of the micelles. Moreover, no elongation or contraction of polymer beads itself were required to induce a change in photoluminescence color as for their PLA beads different form polymer blend comprising mechanosensing dyes inside the host polymers.

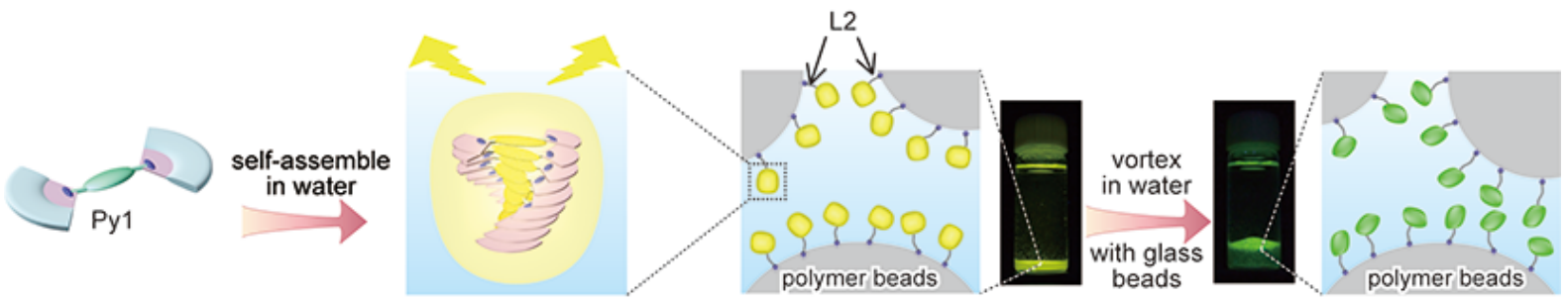

Figure 11. Schematic illustration of polylactic acid (PLA) beads with mechanoresponsive luminescent micelles on the surface and change of their photoluminescent color unpon application of a mechanical force. The pictures were taken under excitation with ultraviolet light. Adapted with permission from ref. [59]. Copyright 2014 American Chemical Society.

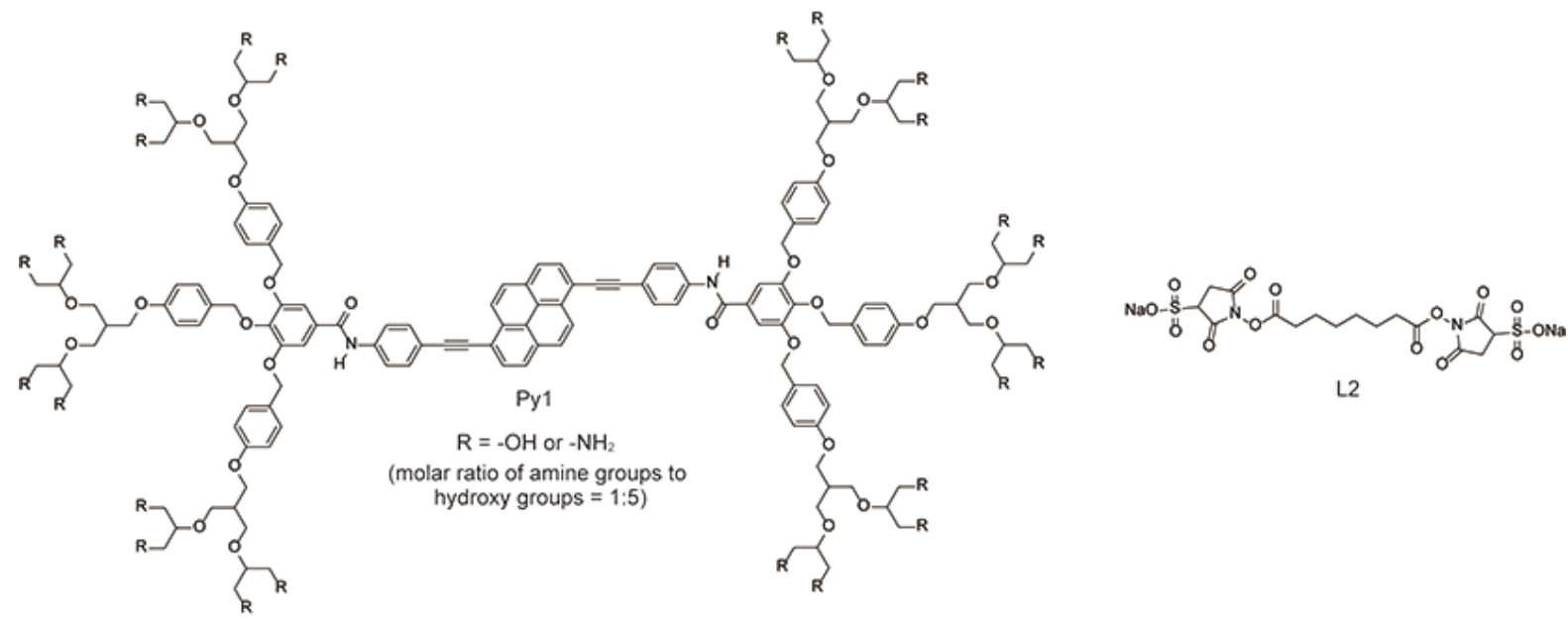

Figure 12. Molecular structure of pyrene-based amphiphile Py1 and water-soluble cross-linker L2.

Mechanically responsive $\pi$ - $\pi$ interactions have also been utilized in supramolecular polymers, where $\pi-\pi$ stacking between electronically complementary (i.e., electron-rich and electron-poor) $\pi$ conjugated groups has been utilized to achieve supramolecular polymerization.[60-63] For example, pairs of naphthalene diimide groups that were introduced into the backbone and termini of polymer P1 (Figure 13) bind to pyrene moieties that were introduced to both ends of polymer P2. Combination of $\mathbf{P 1}$ and $\mathbf{P 2}$ leads to the formation of a supramolecular network structure, in which cross-links are based on intermolecular interactions between electron donor and acceptor groups.[61] The formation of 
donor-acceptor stacked structures is not only evidenced through the material's mechanical properties, but can also be inferred from the appearance of a characteristic reddish color, which arises from the naphthalene diimide / pyrene charge transfer interactions. The materials system at hand was designed to be healable after being damaged. Indeed, after breaking samples of the P1/P2 mixture, the defect could be healed and the tensile modulus could be completely restored by heating for $5 \mathrm{~min}$ to $50{ }^{\circ} \mathrm{C}$. The observed healing efficiency was maintained over several cycles of breaking and heating. Therefore, the $\pi-\pi$ stacking moieties composed of donor and acceptor groups are regarded as reversible units in polymeric materials. While the authors did not exploit this color change for mechanochemical purposes, one can easily foresee that such systems would be prone to color change upon mechanochemical activation and that adequately connected charge-transfer complexes in general should yield similar mechanochromic behavior.

a)
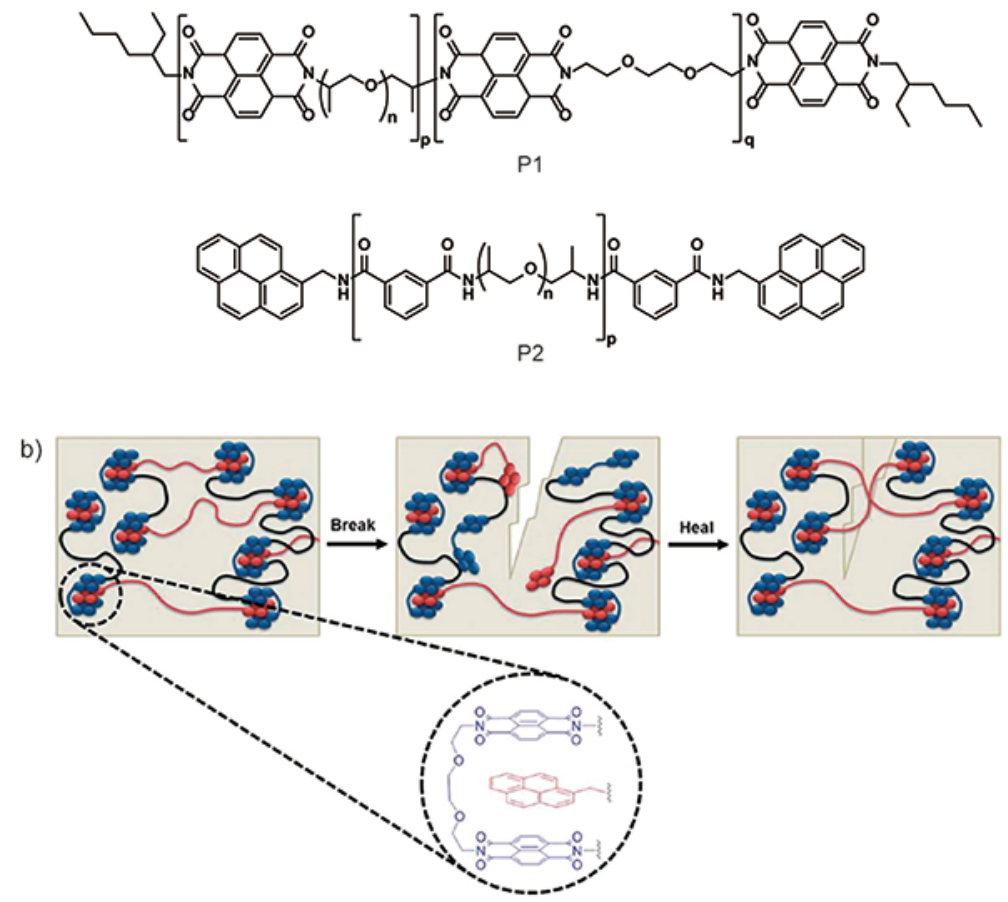

Figure 13. a) Molecular structures of $\mathbf{P 1}$ and $\mathbf{P 2}$, building blocks that were used to assemble a healable, mechanochromic supramolecular polymer. b) Schematic illustration of the healable supramolecular polymers using donor-acceptor interactions between P1 and P2. Panel b reprinted with permission from ref. [63]. Copyright 2014 The Royal Society of Chemistry.

\section{Hydrogen-bonds}

This section focuses on supramolecular polymers based on complementary hydrogen-bonding motifs that exhibit association-dissociation in response to mechanical stimuli. Hydrogen bonds are one of the most commonly utilized types of intermolecular interaction in the fabrication of supramolecular polymers because of their directional character and the ideal strength.[64-67] Furthermore, the associative character of a given supramolecular motif can be fine-tuned by controlling the number of hydrogen-bonding sites. The inherent reversible nature of hydrogen bonds bestows supramolecular polymers made from such motifs with adaptive characters such as thermo-, photo-, chemo- and mechanoresponsive properties.[64-69] In the last 15 years, a large number of hydrogen-bonding supramolecular polymers has been investigated.[70, 71] Interestingly, however, only few reports have described mechanically active hydrogen bonds in supramolecular polymers with unambiguous experimental evidence that show dissociation and formation of hydrogen bonds in response to mechanical stimulation.[72-75] 
a)

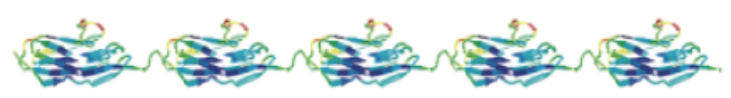

b)

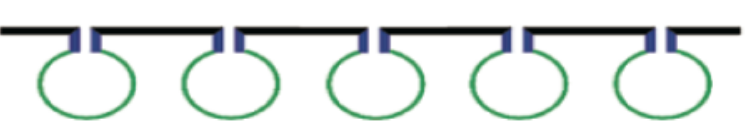

Figure 13. Concept of biomimetic modular polymer design. a) A small section of titin, which has 200-300 repeating immunoglobulin (Ig) domains. b) The design of modular polymer containing multiple loops held by secondary forces. Reprinted with permission from ref. [72]. Copyright 2004 American Chemical Society.

a)

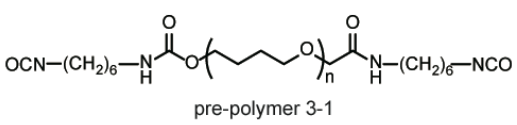

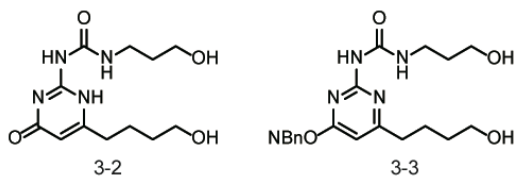

b)

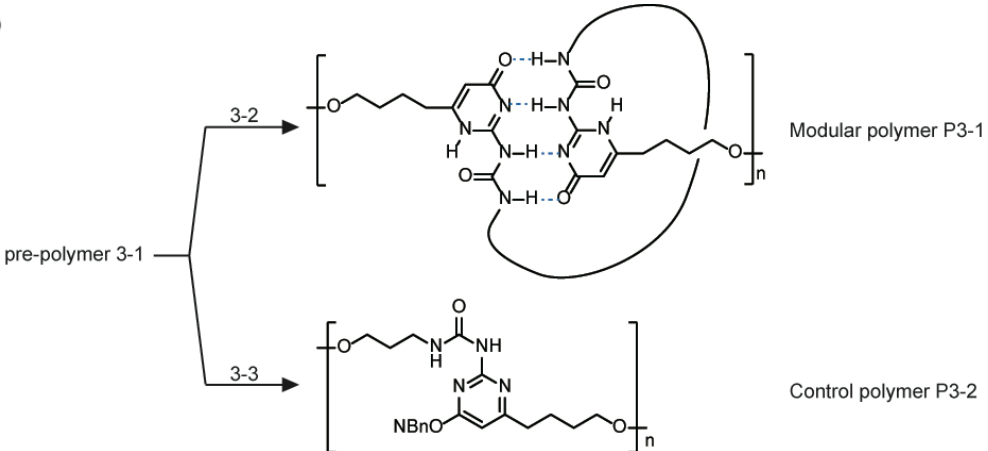

Figure 14. a) Molecular structures of pre-polymer 3-1, free UPy monomer 3-2, and protected UPy monomer 3-3. b) Scheme illustrating the synthesis of P3-1 and P3-2.

a)

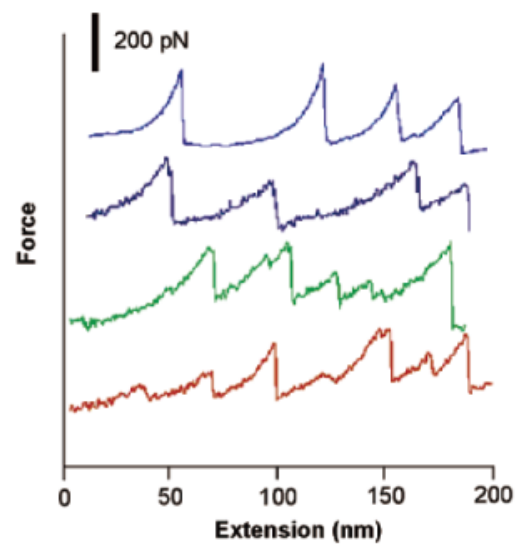

b)

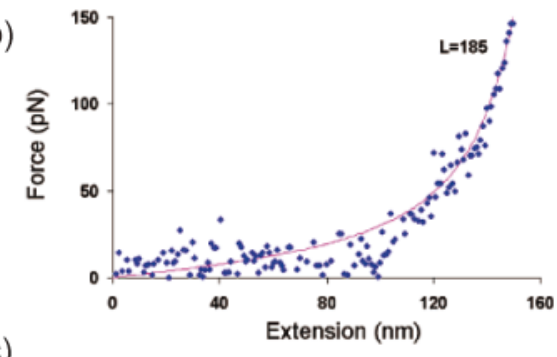

c)

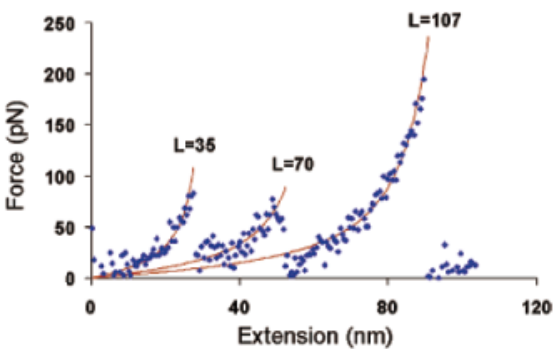

Figure 15. AFM single chain force-extension data. Both the modular polymer P3-1 and the control polymer P3-2 were subjected to single-chain force-extension studies in toluene. a) An overlay of several force-extension curves for modular polymer P3-1. b) One representative single chain force-extension curve for the control polymer P3-2. c) A typical singlechain force-extension curve for the modular polymer P3-1. In b and c, all scattered dots represent experimental data and the solid lines are fitting results. Reprinted with permission from ref. [72]. Copyright 2004 American Chemical Society.

Some of the most compelling examples of mechanoactive hydrogen bonds in polymers have been inspired by the natural protein titin.[72] Titin is a giant protein of muscle sarcomeres and has 200-300 repeating modules that are unfolded sequentially when a mechanical force is applied (Figure 13a), leading to a saw-tooth pattern in the force-extension curve. Each peak is ascribed to individual unfolding events of the repeating modules and reflects the constant force required to unfold each one of them. Using a biomimetic design, a modular polymer having multiple loops (Figure 13b) was developed by the Guan group. Module polymer P3-1 featuring the quadruple-hydrogen-bonding motif 
2-ureido-4[1H]-pyrimidinone (UPy), was prepared using pre-polymer 3-1 and free UPy monomer 3-2 (Figure 14). Control polymer P3-2 was also synthesized using pre-polymer 3-1 and protected UPy monomer 3-3. Single-molecule force-extension studies using atomic force microscopy were carried out for both modular polymer P3-1 and control polymer P3-2. A distinct saw-tooth pattern appeared in the force-extension curve for modular polymer P3-1 (Figure 15a), while the force-extension curves, for control polymer P3-2 did not show the saw-tooth character. Instead, only one peak was observed, which is typical for the entropic extension of a random coil chain. These observations indicate that sequential unfolding of UPy dimers in the modular polymer P3-1 occurs along the polymer chain during single-molecule force-extension studies. When the modular polymer chain is gently pulled, the force increases until the UPy dimers can no longer withstand the load. As a result, the quadruple hydrogen bonding between two UPy moieties yields, the loop opens and extends the chain length of the modular polymer. Therefore, the peak force shown in Figure 15 represents the force required to disassemble the UPy dimers, and the gaps between two peaks are characteristic of the unfolding of the extra slack provided by the loop following dimer dissociation. Since the UPy dimers can be connected in different ways during the synthesis, the force needed to deform the dimer varied between $c a$. 200 $\mathrm{pN}$ for the preferred head-to-head arrangement vs. $c a .100 \mathrm{pN}$ for the less favorable head-to-tail or tailto-tail connectivity. Additionally, the force-extension curve showed different length between force peaks due to the chain length distribution of the pre-polymer 3-1.

To overcome several limitations of the modular polymer P3-1, a newly designed monomer 3-4 that forms quadruple hydrogen bonds was subsequently prepared by Guan and coworkers using peptidomimetic $\beta$-sheet motif (Figure 16a).[73] Two loops were used to promote proper intrachain dimerization, thereby limiting dimerization with a nonadjacent unit within the same polymer or with units in different polymer chains. Alkyl chains were used as the loop-forming unit in lieu of polydisperse poly(tetramethyleneoxide), which led to constant gaps between force peaks in the forceextension curve. The double-closed loop (DCL) polymer P3-3 (Figure 16b) was obtained by reacting equimolar amounts of 4,4'-methylenebis-(phenyl-isocyanate) (MDI) and the DCL monomer. Singlemolecule force-extension studies using atomic force microscopy were also carried out for the DCL polymer (Figure 17). Compared to the modular polymer P3-1, the saw-tooth pattern showed greater uniformity. The force required to break each module was, however, smaller $(\sim 50 \mathrm{pN})$, which was explained by the smaller dimerization constant of the peptidomimetic $\beta$-sheet units $\left(\sim 10^{4}\right)$ compared to that of UPy dimers $\left(\sim 10^{7}\right)$. Despite the increased uniformity, a distribution of the contour length $(\Delta L)$ between consecutive peaks in the force-extension curves was observed $(\Delta L \approx 5-21 \mathrm{~nm})$. This phenomenon was thought to arise from $\pi-\pi$ stacking between dimerizing units. The association energy between two dimerizing modules is comparable to that of the four hydrogen bonds in each dimerizing module. Therefore, during the polymer extension, the four hydrogen bonds in each module as well as $\pi-\pi$ stacking between modules dissociate simultaneously.
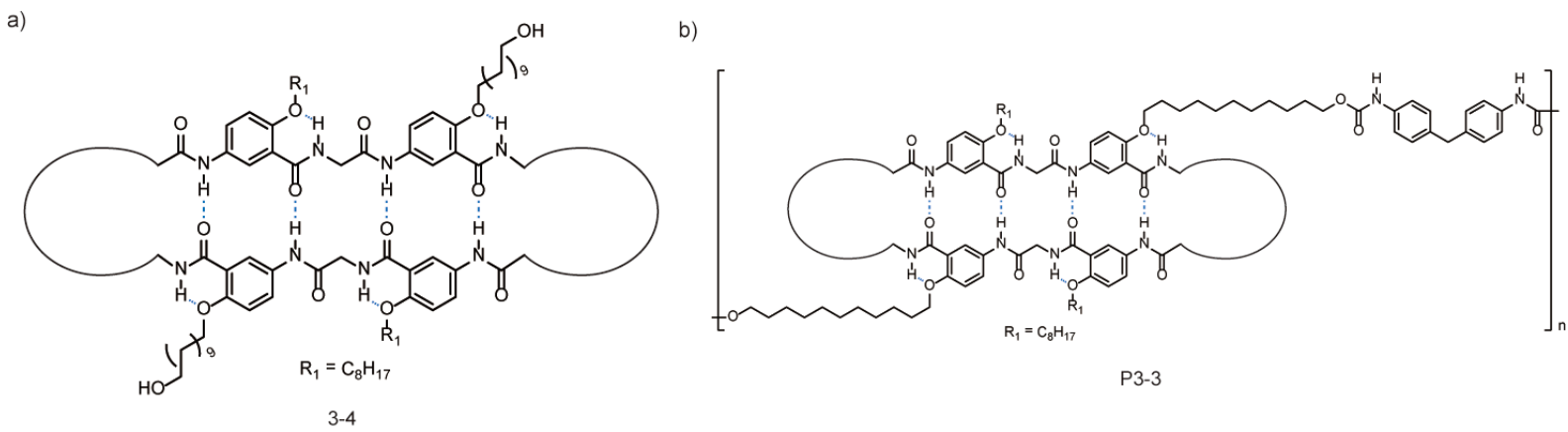

Figure 16. Molecular structures of a) double-closed loop monomer 3-4 and b) the resulting polymer P3-3. 


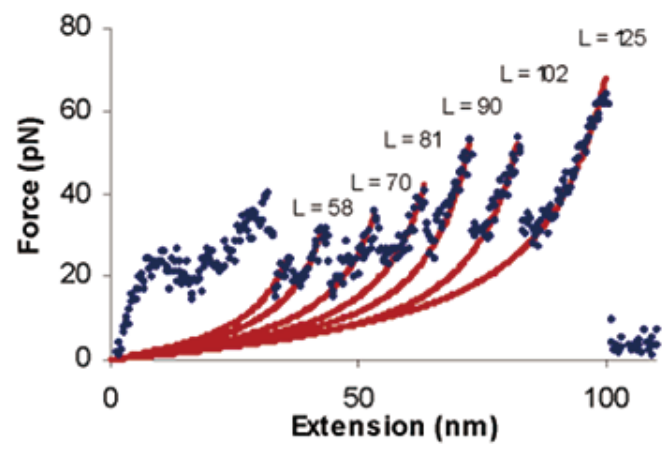

Figure 17. AFM single-molecule force-extension curves for the DCL modular polymer shown in Figure 16b. Reprinted with permission from ref. [73]. Copyright 2004 American Chemical Society.

Taking into account the knowledge obtained from both modular systems, Guan and coworkers prepared UPy-based cross-linker 3-5 having two alkyl chain loops (Figure 18a) in the module unit.[74] The authors expected that the mechanical properties of the resulting cross-linked polymers would be significantly enhanced because of the reversible nature of the UPy-based module and strength of quadruple hydrogen bonds in each module. Cross-linked poly( $n$-butyl acrylate) was chosen as the backbone, because of its elastomeric character. Poly(ethylene glycol) dimethacrylate was chosen as the control cross-linker. Several films were prepared by copolymerization of $n$-butyl acrylate and cross-linker 3-5 or poly(ethylene glycol) dimethacrylate. Figure 28b shows the stress-strain curves for samples cross-linked with $6 \mathrm{~mol} \%$ of either the cross-linker 3-5 or the poly(ethylene glycol) dimethacrylate control. Upon cross-linking with 3-5, both modulus and tensile strength substantially increased without sacrificing the maximum extensibility. By contrast, the covalently cross-linked control suffered from a much smaller strain at break. The striking difference between the two materials was attributed to the increased energy dissipation ability of cross-linker 3-5.

a)

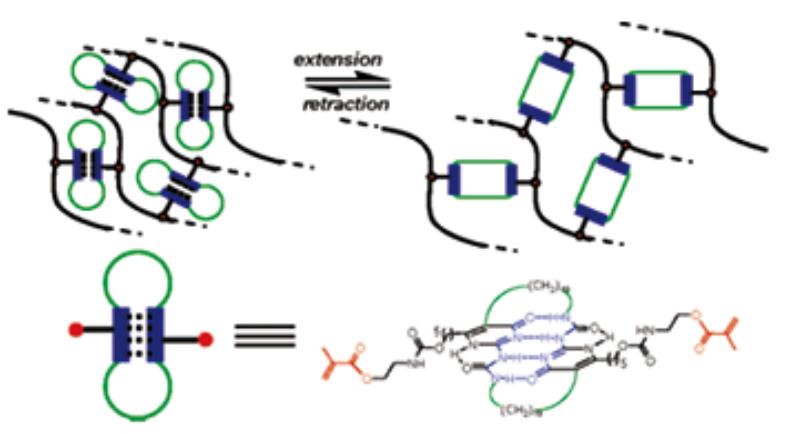

b)

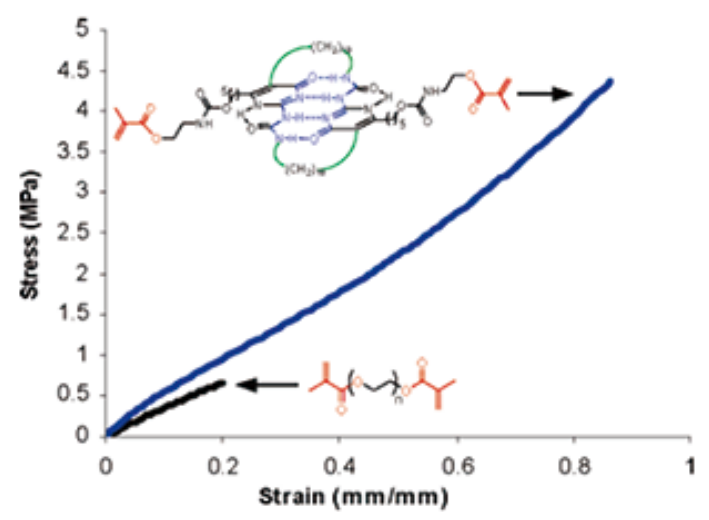

Figure 18. a) Schematic representation of the design of a biomimetic modular cross-linker. b) Stress-strain curves for $6 \%$ cross-linked poly( $n$-butyl acrylate) rubber for the sample (blue) and control specimen (strain rate: $100 \mathrm{~mm} / \mathrm{min}$, at room temperature). Reprinted with permission from ref. [74]. Copyright 2007 American Chemical Society.

The same group also developed linear polymer P3-4 using UPy-based double closed-loop modules (Figure 19).[75] To avoid undesired hydrogen-bonding and prevent complicated folding structures, the polymer did not possess any hydrogen-bonding sites other than the UPy moieties in each module. Linear module polymer P3-4 and control polymer P3-5 were obtained by acyclic diene metathesis using corresponding monomers. In control polymer P3-5, an $o$-nitrobenzyl (NBn) protection group was introduced, in order to suppress hydrogen-bond formation in each module. As shown in Figure 19a, the stress-strain curves of P3-4 and P3-5 are completely different from each other. Control polymer P3-5 fractures at 7\% strain. By contrast, modular polymer P3-4 exhibited large deformation with a maximal strain of $>100 \%$. At low strains, P3-4 is quite stiff and showed a relative high Young's modulus. After 5\% elongation, P3-4 exhibited a large deformation with modest increase in stress, which means that large amount of energy is absorbed as a result of the sequential unfolding 
a)

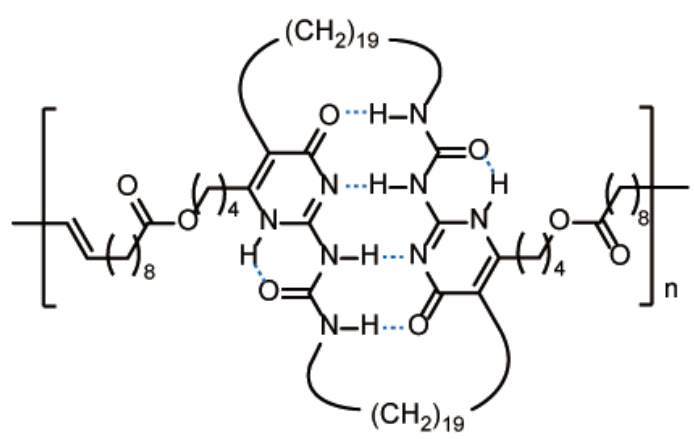

P3-4

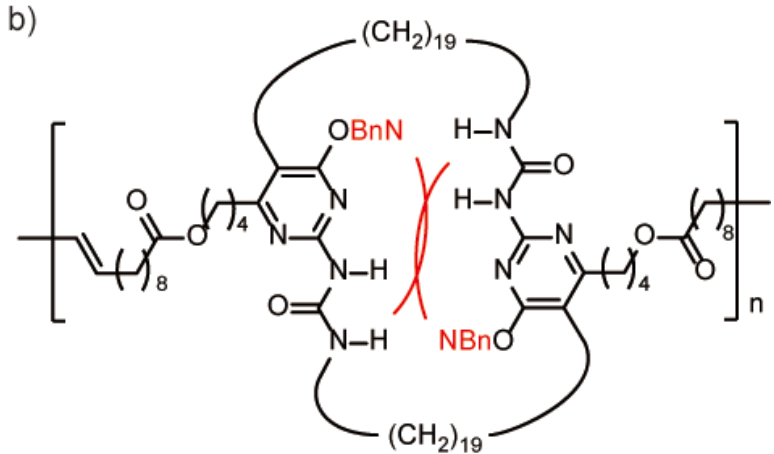

P3-5

Figure 19. Molecular structure of a) linear double-closed loop module polymer P3-4 and b) control polymer P3-5.

The shape-memory properties of the linear polymer P3-4 and its mechanical behavior have been also investigated in a later study. After 10 loading-unloading cycles, the polymer was set at $135 \%$ of its original length. By leaving this sample at room temperature for $18 \mathrm{~h}, \mathbf{P 3}-\mathbf{4}$ recovered up to $110 \%$ of its original length (Figure 20). The complete recovery of original mechanical properties of P3-4 was achieved by heating the sample at $\sim 80{ }^{\circ} \mathrm{C}$ for $\sim 30 \mathrm{~s}$ after loading-unloading cycles. Moreover, when the sample was heated and elongated to $250 \%$ strain and then cooled to $5^{\circ} \mathrm{C}$, the temporary shape was fixed. A gradual temperature increase let to sample retraction and further heating resulted in complete shape recovery of P3-4 film. The observed stress-strain responses and the shape-memory characters of P3-4 are derived from the dynamic nature of UPy dimerization, as further corroborated by the absence of similar properties for control polymer P3-5. The mechanism to explain the observed phenomena is illustrated by the authors (Figure 20). UPy homodimers are unfolded upon mechanical or thermal stimulation. When the stimulus is removed, UPy inter chain cross-dimers form in the polymer films. Subsequent heating induces recombination of UPy moieties and lead to the recovery of the original shape and mechanical properties.

a)

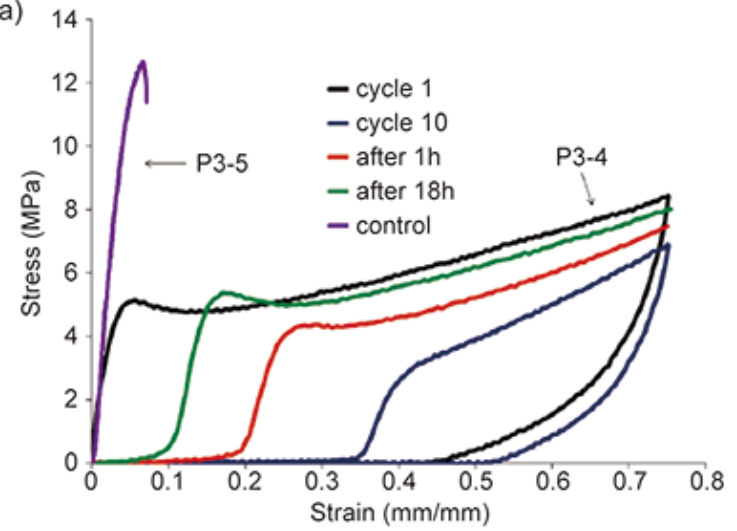

b)

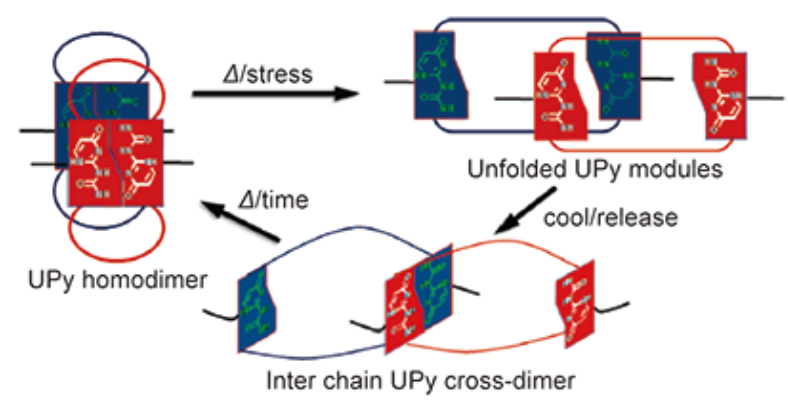

Figure 20. a) Stress-strain curves for polymer P3-4 and control P3-5 shown in Figure 19. b) Proposed molecular mechanism for shape-memory behavior. Adapted with permission from ref. [75]. Copyright 2009 American Chemical Society.

\section{Metal-ligand interactions}

Metal-ligand interactions represent another class of non-covalent supramolecular motifs, which have been shown to be very useful in the context of designing stimuli-responsive supramolecular polymers. The motif type represents great versatility, as the strength of the interaction between a polymer-bound ligand and metal ions, the responsiveness to various stimuli, and the response can be varied over a broad range via the variation of both metal ion and ligand. A major advantage of metallo- 
supramolecular polymers is the possibility of combining the processability of polymers, while retaining some of the unique properties of the metal centers. In 2003, a seminal contribution of Rowan and coworkers introduced "multistimuli, multiresponsive metallo-supramolecular polymers".[76] The material introduced by the Rowan group consisted of a polyethyleneglycol (PEG) backbone which was functionalized at both ends with a bis(2,6-bis(1'-methyl-benzimidazolyl)-4-hydroxypyridine) ligand (Mebip; Figure 21). Such building blocks can be used to construct longer polymer chains by adding various metal ions in the form of weakly coordinating salts, such as nitrates, perchlorates, or bistriflimides. Chain extension is achieved by stoichiometric complexation of the metal by the polymer-bound ligands. Of course, the nature of the metal ion plays a pivotal role in the overall molecular architecture of the materials. In the most common case, i.e. where ditopic polymeric ligands are used, the metal center determines if linear connections (as in the case of bivalent ions) or branching/network points are formed (as in the case of trivalent ions), since they are coordinated by two or three telechelic ligands. Many properties of the resulting material can be adjusted by carefully choosing the metal ion. For instance, luminescent europium ions may be used as internal probe indicating the status of the metal-ligand bond, since they exhibit luminescence through an "antenna effect", i.e. that energy initially absorbed by the ligand is channeled to the metal through a metal-toligand charge transfer.[77, 78]
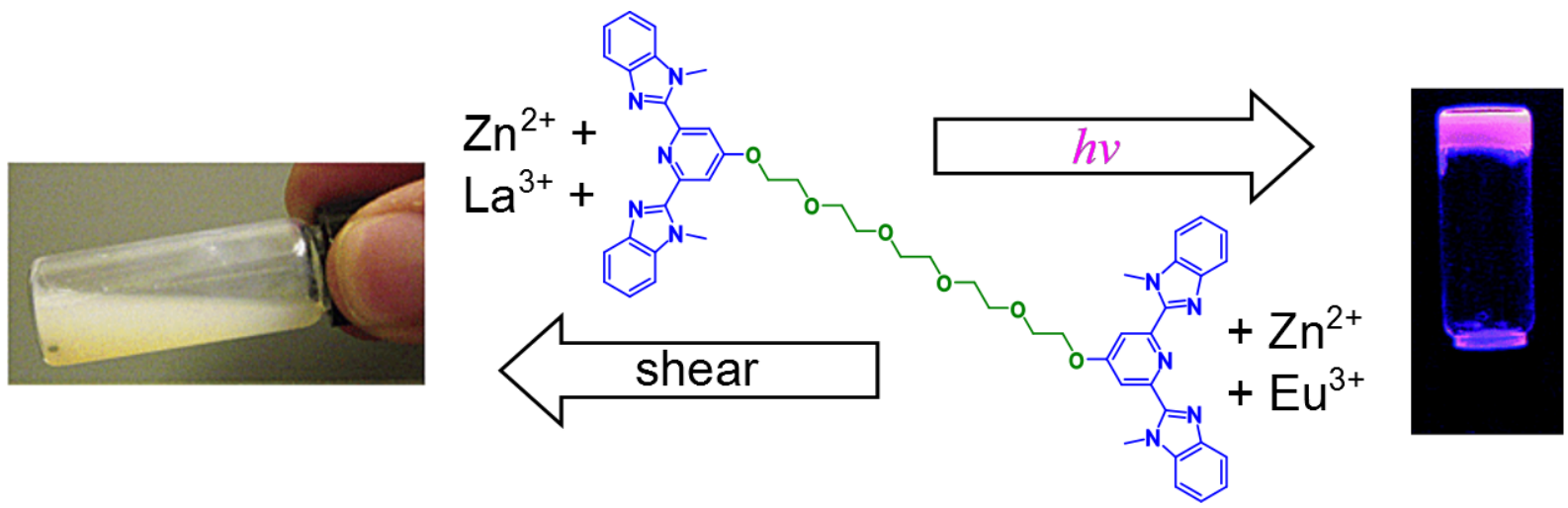

Figure 21. Illustration of chemical structure, mechanical response, and optical characteristics of multi-stimuli responsive metallo-supramolecular polymers. Adapted with permission of ref. [76]. Copyright 2003 American Chemical Society.

While metallo-supramolecular polymers had been previously studied,[79] the work of Beck et al. opened new avenues by demonstrating that metal-ligand bonds could be mechanically activated,[76] although the materials studied were gels, and thus not sensu stricto solid materials due to presence of solvent (here acetonitrile). The mechanical activation of weak coordination bonds was demonstrated in metallo-supramolecular gels containing the telechelic building block shown in Figure 21 and $\mathrm{Zn}^{2+}: \mathrm{La}^{3+}$ (ratio 97:3) through the characteristic thixotropic (shear-thinning) behavior of the gels. Upon manual agitation, stable gels were rapidly liquefied as a result of the disengagement of the ligands from the metal ions. When the system was allowed to rest, stable gels were reformed. The behavior was shown to depend upon the amount of solvent present. Furthermore, thermal stimuli-responsiveness was demonstrated, and the lanthanide ions were shown to separate from the ligands more easily than the transition metal ions. Additionally, responsiveness to chemical stimuli, such as a competing ligand (i.e. formate anion, which binds preferentially to the $\mathrm{Eu}^{3+}$ ions in comparison to the Mebip ligands) was also demonstrated. On account of the supramolecular nature of the binding motifs, all stimuli, i.e. mechanical, thermal, and chemical, could be used to reversibly dissociate the metallopolymers studied.

A broad range of stimuli-responsive metallopolymers based on Mebip-terminated macromonomer-metal ion interactions has in the meantime been investigated, not only with respect to the mechanical activation of the weak links, but also in terms kinetic formation of the metal-ligand complex,[80] influence of the metal centers (dynamic vs. inert, two- vs. three functional, variation of optical properties) and polymer backbones (e.g. polar vs. nonpolar, rubbery vs. glassy $v s$. semicrystalline). It is noted that in solid materials, the morphology has a great influence on the mechanical properties and the underlying stress-transfer processes of these materials.[81] While mechanically induced disassembly of the metal-ligand motifs has in some cases been inferred, this 
process has rarely been experimentally supported. For example, the groups of Weder and Rowan demonstrated that optical healing can be achieved in metallo-supramolecular polymers assembled from a rubbery telechelic poly(ethylene-co-butylene) (15) that was chain-terminated with 2,6-bis(1'methylbenzimidazolyl)-pyridine (Mebip) ligands and $\mathrm{Zn}\left(\mathrm{NTf}_{2}\right)_{2}$ or $\mathrm{La}\left(\mathrm{NTf}_{2}\right)_{3}$ to afford $\mathbf{1 5} \cdot \mathrm{Zn}\left(\mathrm{NTf}_{2}\right)_{2}$ and 15. $\mathrm{La}\left(\mathrm{NTf}_{2}\right)_{3}$ (Figure 22).[82] The metal-ligand binding is dynamic (more so in the case of $\mathrm{La}^{3+}$ than $\mathrm{Zn}^{2+}$ ) and the polymers can be depolymerized upon exposure to certain external stimuli such as light, heat, and presumably also mechanical forced. Small-angle X-ray diffraction experiments and electron microscopy and revealed that these metallo-upramolecular materials micro-phase separate into lamellar structures, in which the metal-ligand complexes form a "hard" phase, which serves to physically crosslink the "soft" phase formed by the poly(ethylene-co-butylene) cores. The Mebip ligands and Mebip/metal complexes absorb ultraviolet (UV) light and exhibit a low fluorescence quantum yield. Thus, when the materials are irradiated with UV light, the absorbed energy is converted to heat. This results in the temporary disengagement of the metal-complexes, or in other words, depolymerization of the metallopolymer under formation of a low-viscosity melt. Upon removal of the light source, the metal-ligand complexes reform and the material's original properties are restored. This transformation allows for optical healing of (mechanically induced) damages areas of the material. When deliberately damaged films of $15 \cdot \mathrm{Zn}\left(\mathrm{NTf}_{2}\right)_{2}$ or $15 \cdot \mathrm{La}\left(\mathrm{NTf}_{2}\right)_{3}$ were exposed to UV light with an intensity of $\sim 950 \mathrm{~mW} / \mathrm{cm}^{2}$, the defects were repaired in less than 1 minute. Mechanical analysis showed that the original mechanical properties of the materials could be fully restored and it was shown that the materials can be repeatedly damaged and healed. It was shown that under comparable conditions films of $\mathbf{1 5} \cdot\left[\mathrm{La}\left(\mathrm{NTf}_{2}\right)_{3}\right]$ healed more efficiently than films made from $\mathbf{1 5} \cdot\left[\mathrm{Zn}\left(\mathrm{NTf}_{2}\right)_{2}\right]$, consistent with the lower stability of $\mathrm{La}^{3+} /$ Mebip complexes vis-à-vis the $\mathrm{Zn}^{2+} /$ Mebipbased counterparts and a higher level of light-induced depolymerization of the former. The concept of photo-thermal induced healing of supramolecular materials is applicable to any supramolecular polymer with a binding motif that is sufficiently dynamic and the concept has now been utilized in a variety of materials systems[83-85].
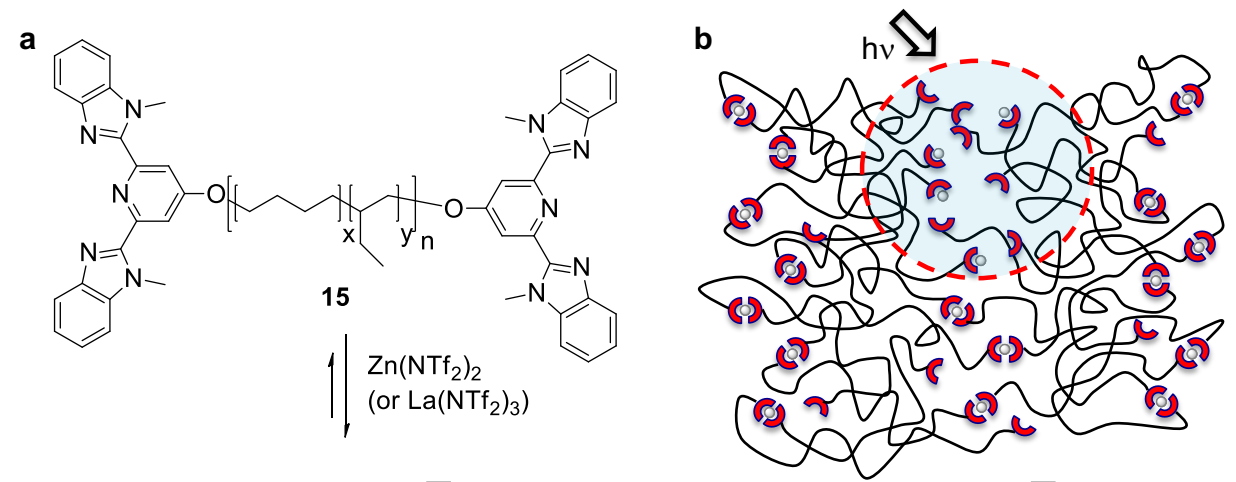

C

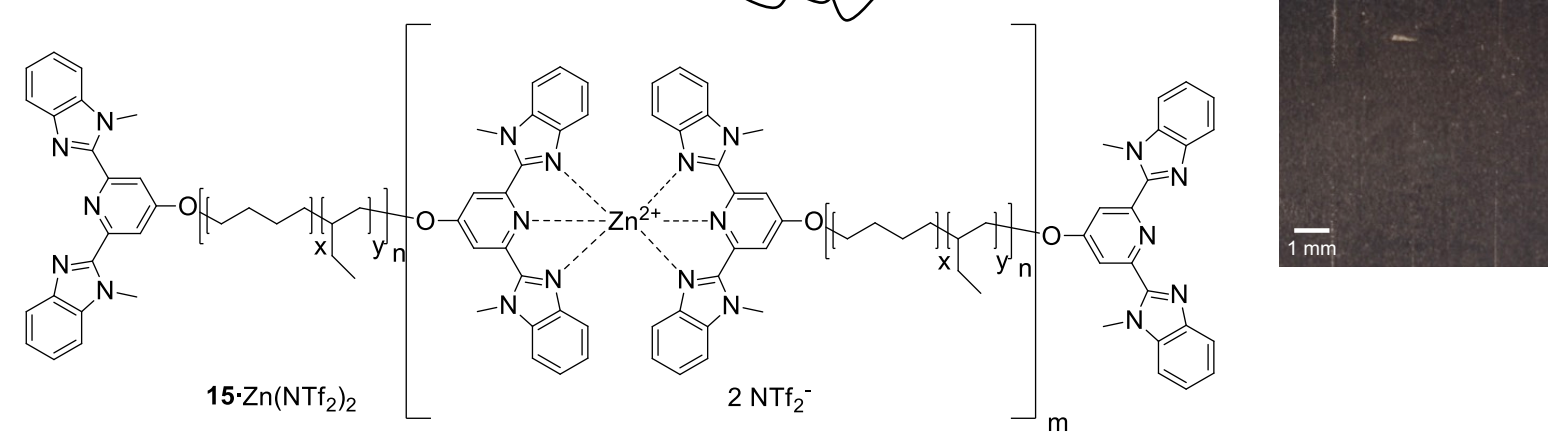

Figure 22. a) Chemical structure of macromonomer 15 and the metallo-supramolecular polymers $15 \cdot\left[\mathrm{M}(\mathrm{NTf})_{\mathrm{v}}\right]$ where $\mathrm{M}=$ $\mathrm{Zn}^{2+}(\mathrm{v}=2)$ or $\mathrm{M}=\mathrm{La}^{3+}(\mathrm{v}=3)$. b) Schematic representation of the lamellar structure of $\mathbf{1 5} \cdot\left[\mathrm{M}\left(\mathrm{NTf}_{2}\right)_{\mathrm{v}}\right]$. c) Image illustrating the optical healing of a film of $\mathbf{1 5} \cdot\left[\mathrm{Zn}\left(\mathrm{NTf}_{2}\right)_{2}\right]\left(\right.$ ratio of $\left.\mathbf{1 5}: \mathrm{Zn}\left(\mathrm{NTf}_{2}\right)_{2}=1: 0.7\right)$ with UV light $\left(\lambda=320-390 \mathrm{~nm}, 950 \mathrm{~mW} / \mathrm{cm}^{2}\right.$, $2 \times 30 \mathrm{sec})$. The figures are adapted from [82].

The repeated healability of the metallo-supramolecular polymers discussed above implies that on the molecular level, mechanical damage exclusively causes (reversible) disassembly of the metal-ligand 
motifs, as shown schematically in Figure 22, and not cleavage of covalent bonds. However, until recently, this mechanism had not been confirmed. With the objective to probe the mechanistic aspects of mechanotransduction in such materials, the Weder group recently embarked on the investigation of mechanically-induced responses of metallo-supramolecular networks. Their study showed that metallo-supramolecular polymers based on macromonomer 5 (a telechelic poly(ethylene-co-butylene) with Mebip ligands at the termini) and the europium salt $\mathrm{Eu}\left(\mathrm{ClO}_{4}\right)_{3}$ (Figure 23a) are mechanoresponsive. $[86,87] \mathrm{The}^{3+}$ :Mebip complexes serve both as mechanically responsive binding motifs and built-in optical probes that can monitor the extent of (dis)assembly due to their characteristic photoluminescent properties. Dose-dependent and reversible metal-ligand dissociation occurs upon exposure to ultrasound in solution (Figure 23b, 24a). The absence of ultrasound-induced dissociation of a low-molecular weight model complex and in-depth studies of temperature effects confirm that the dissociation is indeed the result of mechanical activation (for extensive review on sonochemical activation please refer to $[11,88])$. The influence of the strength of the metal-ligand interactions on the mechanically induced dissociation was also explored. Metallo-supramolecular polymers in which the Mebip ligands were substituted with more strongly coordinating dipicolinate ligands (Figure 23c) do not dissociate upon exposure to ultrasound. Finally, it was shown that mechanochemical transduction in metallo-supramolecular polymers is also possible in the solid state. This permitted the mending of damaged objects through ultrasound (Figure 24c) as well as mechanochromic behavior based on metal-exchange reactions in metallo-supramolecular polymers imbibed with an auxiliary metal salt (Figure 24b). This was achieved by imbibing the metallo-supramolecular polymer with a $\mathrm{Fe}\left(\mathrm{ClO}_{4}\right)_{2}$ solution and subjecting the material thus treated to ultrasound or a macroscopic force. Gratifyingly, no color change occurred by immersion in the salt solution, and only upon mechanochemical activation was an irreversible color change, characteristic of the $\mathrm{Fe}(\mathrm{Mebip})_{2}$ complexes observed (Figure 24b).

a
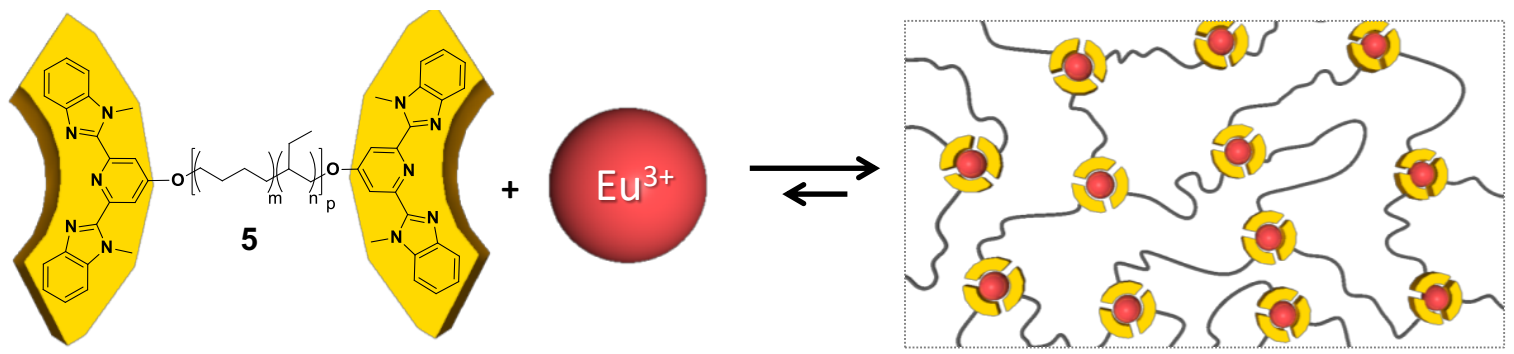

b
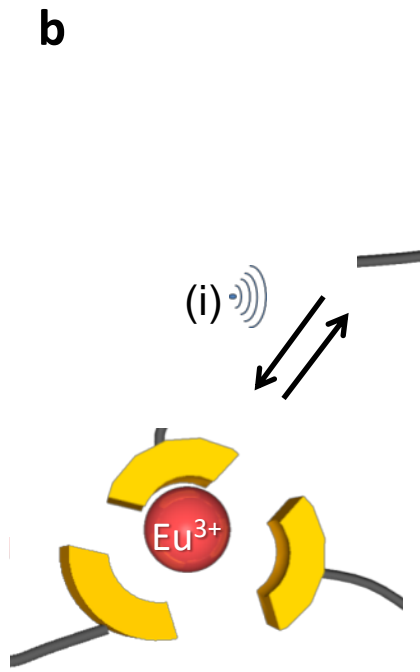

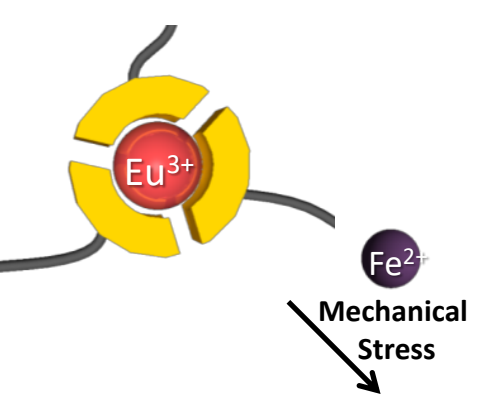

(iii)

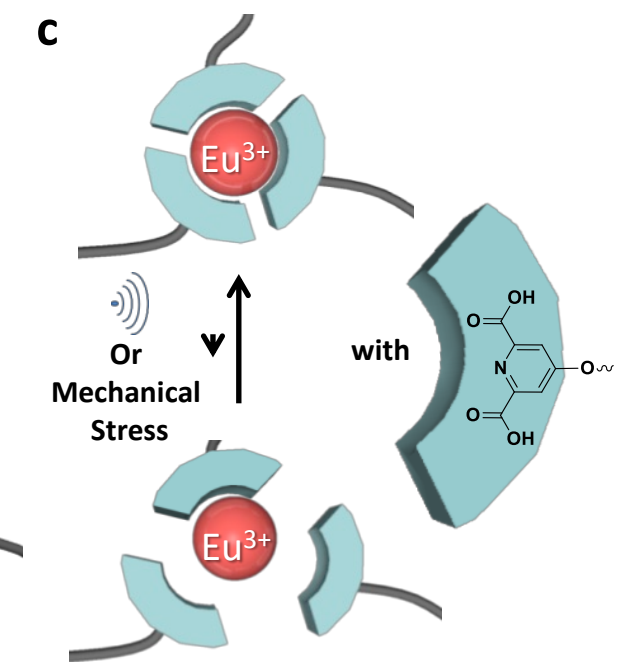

Figure 23. Structure of a mechano-responsive metallo-supramolecular polymer network and schematic representation of (dis)assembly mechanisms. a) Formation of (5) $)_{1.5} \cdot\left[\mathbf{E u}\left(\mathbf{C l O}_{4}\right)_{3}\right]$ networks by assembly of 5 and $\mathrm{Eu}\left(\mathrm{ClO}_{4}\right)_{3} \cdot 6 \mathrm{H}_{2} \mathrm{O}\left(\mathrm{ClO}_{4}{ }^{-}\right.$ counterions are omitted for clarity). b) (i) Reversible dissociation of the $\mathrm{Eu}^{3+}$-ligand complexes upon ultrasonication and irreversible exchange with $\mathrm{Fe}^{2+}$ ions in films of $(\mathbf{5})_{1.5} \cdot\left[\mathbf{E u}\left(\mathbf{C l O}_{4}\right)_{3}\right]$ imbibed with a $\mathrm{Fe}\left(\mathrm{ClO}_{4}\right)_{2}$ solution as a result of (ii) ultrasonication or (iii) other mechanical forces. c) Deprotonated dipicolinic acid ligands bind strongly to $\mathrm{Eu}^{3+}$ and these complexes can hardly be mechanically dissociated (triethylammonium ions are omitted for clarity). Reprinted with permission from ref.[86]. Copyright 2014 American Chemical Society. 
a

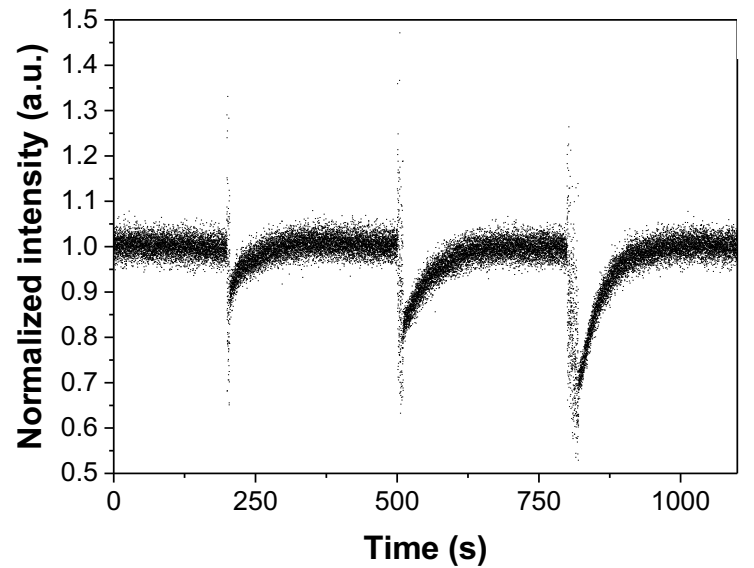

b

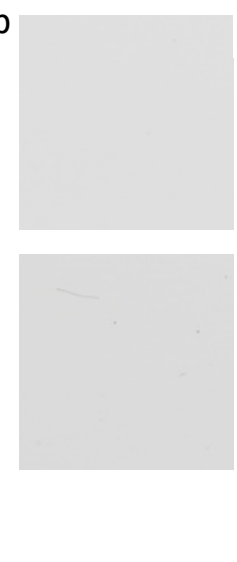

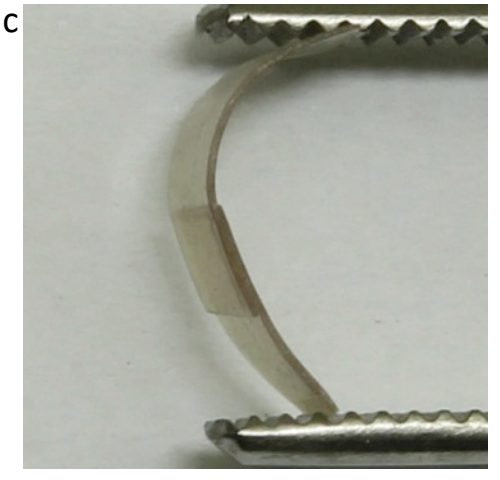

Figure 24. a) Emission of (5) $)_{1.5} \cdot\left[\mathbf{E u}\left(\mathbf{C l O}_{4}\right)_{3}\right]$ in $\mathrm{CHCl}_{3}$ monitored at $620 \mathrm{~nm}$ over time. The solution was subjected to three ultrasonication pulses of $10 \mathrm{~s}$ each. b) Images of $(\mathbf{5})_{1.5} \cdot\left[\mathbf{E u}\left(\mathbf{C l O}_{4}\right)_{3}\right]$ films swollen in an acetonitrile solution of $\mathrm{Fe}(\mathrm{ClO})_{2}$ for $5 \mathrm{~d}$ before (top) and after (bottom) ultrasonication. c) Image of two films of $(\mathbf{5})_{1.5} \cdot\left[\mathbf{E u}\left(\mathbf{C l O}_{4}\right)_{3}\right]$ mended together by ultrasonication. Adapted with permission from ref. [86]. Copyright 2014 American Chemical Society.

In all of the above-summarized studies, the Mebip ligands were covalently attached to a telechelic core and served either to chain-extend or cross-link these building blocks, in connection with various metal ions, into linear or cross-linked metallo-supramolecular polymers. Rowan et al. took a different approach when they blended complexes based on monofunctional dodecyl-Mebip and metal ions into methacrylate-based polymer matrices.[89] Interestingly, when complexes with platinum ions were used, a mechanically responsive material was obtained, which displayed a mechanically induced color change both in absorption and photoluminescence. The mechanically induced optical were related to a variation of the Pt-Pt interactions (i.e., the distances in small Pt-ligand crystallites that formed in the glassy matrix), which was stable after applying mechanical forces in the classy state but could be deliberately reverted by heating above the $T_{\mathrm{g}}$ of the polymer matrix.

The discovery that mechanochemistry can be at play in metal-containing polymers goes back to 2004, when Sijbesma and coworkers demonstrated mechanochemical activation in polymers comprising phosphine ligands that were coordinating late transition metals such as palladium or iridium. Specifically, poly(tetrahydrofuran) (pTHF) with telechelic phosphine moieties was used to complex $\mathrm{PdCl}_{2}$, which resulted in the formation of linear supramolecular polymers and smaller cyclic structures (Figure 25).[90] The Pd-phosphine bonds could be selectively broken upon ultrasonication solutions of the metallopolymer and no by-products could be identified by NMR spectroscopy. Furthermore, if only the weak coordination bond was to be selectively cleaved by sonication, the supramolecular polymer assembly should simply be driven by concentration and the original numberaverage molecular weight should be recovered. Since no irreversible polymer degradation upon multiple cycles of breaking and reformation was observed, one can conclude that the Pd phosphine connection could be reversibly and selectively cleaved. The re-formation of the metallosupramolecular polymer was shown to be much slower than in the previously described Mebip systems (dozens of hours vs. minutes). Ultrasonication was shown to preferentially disrupt longer chains, which is indicative of mechanical activation as a thermal process should affect short and long chains alike. A most interesting feature of the mechanically responsive Pd-containing is the catalytic potential of the metal centers, which can be reversibly activated. This feature was used, for example, to induce the activation of an olefin metathesis catalyst mechanically.[91-94] 


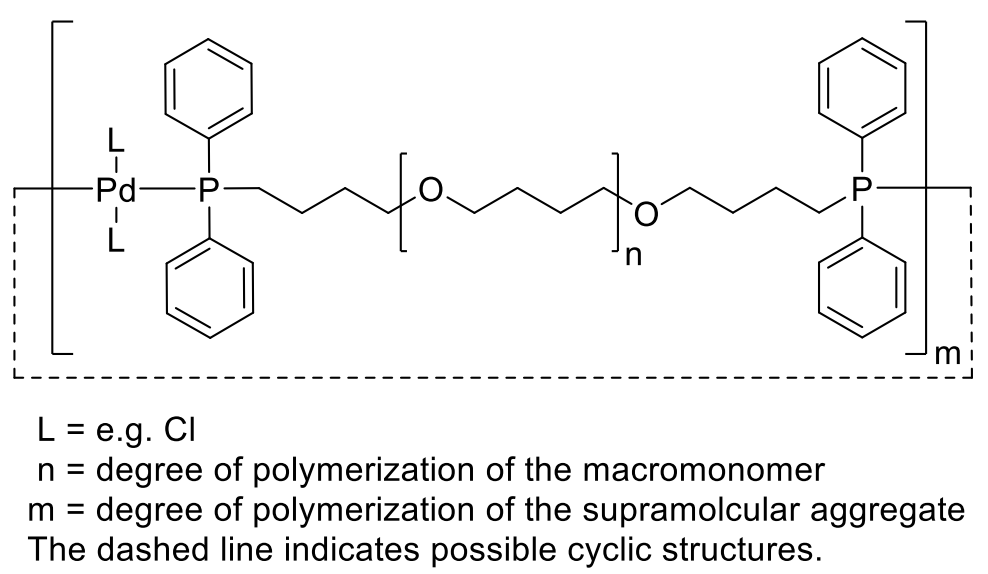

Figure 25. General structure of the mechanically responsive Pd-phosphine containing metallo-supramolecular polymers studied by Sijbesma and co-workers [90].

In a subsequent study, Paulusse et al. quantified the extent of the sonochemically induced dissociation of slightly modified phosphine ligands (cyclohexyl instead of phenyl substituents at the phosphorus) from the Pd centers by adding 1-(dicyclohexylphosphino)dodecane as a competing ligand.[95] The metallopolymer was kinetically stable when it was mixed in solution with the alkyldiphenylphosphine, since exchanges within the Pd coordination sphere are very slow. However, upon ultrasonication, a significant and irreversible reduction of the metallopolymer's molecular weight was observable, on account of mechanically induced dissociation of the metallopolymer and subsequent irreversible binding of 1-(dicyclohexylphosphino)dodecane to the free Pd. The study revealed that the dissociation rate of the current system upon ultrasonication was too slow for a practical application in catalysis.

Similar metallopolymers based on diphenylphosphine-terminated macromonomers in combination with $\mathrm{Rh}^{+}$or $\mathrm{Ir}^{+}$ions resulted in the formation of gel-like networks when the building blocks were combined in a solvent such as dichloromethane or chloroform.[96] These gels could be liquefied upon ultrasonication (Figure 26), as a result of ligand exchanges that preserved the coordination stoichiometry of the metal centers while reducing the number of cross-links. After sonication, the equilibrium network re-formed almost immediately in the case of $\mathrm{Rh}^{+}$(gel phase after 1 hour at $+20{ }^{\circ} \mathrm{C}$ ), whereas the process was much slower in the case of the $\operatorname{Ir}^{+}$based gel (sol phase present after 3 days at $-20^{\circ} \mathrm{C}$ ).
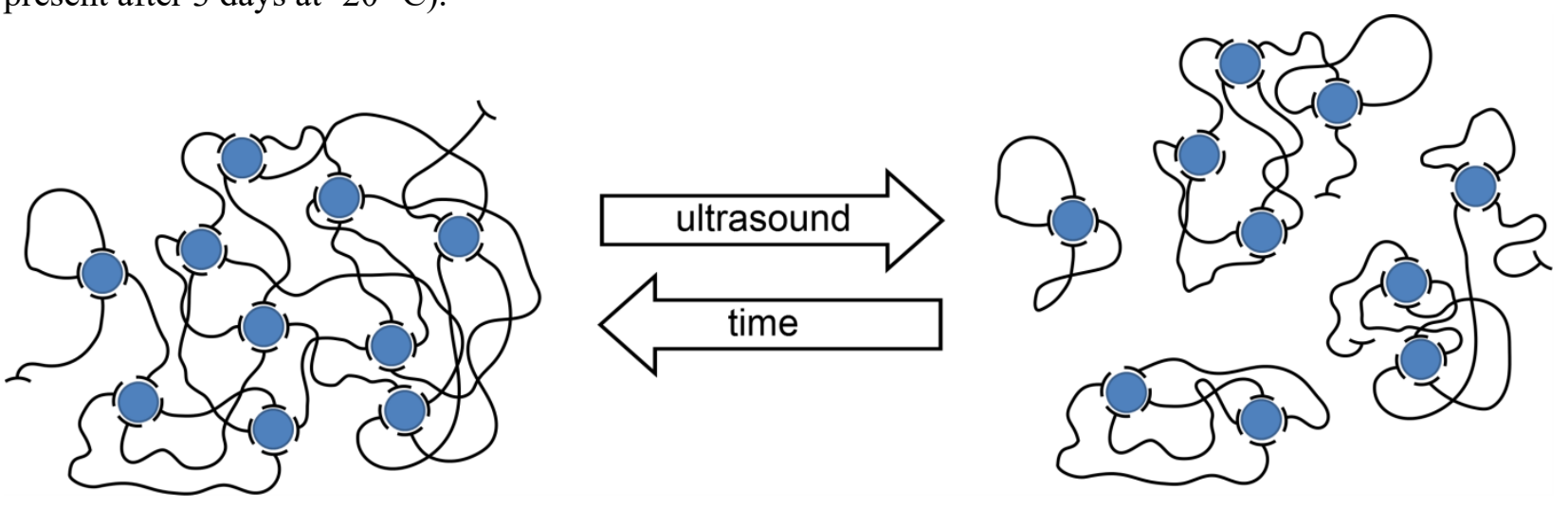

Figure 26. Proposed mechanism for the liquification of phosphine-Rh or phosphine-Ir supramolecular gels upon ultrasonication [96].

Through the study of "mixed" metallopolymers based on $\mathrm{Pd}^{2+}$ and $\mathrm{Pt}^{2+}$ salts and phosphineterminated telechelic macromonomers, Sijbesma and coworkers were able to address the question how selective ultrasonication induced chain scission of metallopolymers can be.[97] Moore and coworkers had previously reported that selectivity in chain scission requires the bond energy of the "weak links" - also referred to as mechanophores - is approximately $100-125 \mathrm{~kJ} \cdot \mathrm{mol}^{-1}$ lower than that of the other (residual) bonds.[98] The hybrid system containing both $\mathrm{Pd}^{2+}$ and $\mathrm{Pt}^{2+}$ metal ions focused on very 
small bond energy differences; according to density functional theory calculations the complex formed between $\mathrm{PtCl}_{2}$ and pentyl-di-cyclohexylphosphine features a metal-P bond that is $c a .30 \mathrm{~kJ} \cdot \mathrm{mol}^{-1}$ stronger than the corresponding Pd complex. While such a small difference in coordination strength should preclude complete selectivity, ultrasonication experiments of solutions of the metallopolymer demonstrated that chain scission at the Pd centers was - especially in the initial stages of ultrasonication -favored over chain scission at Pt centers. Similarly,[97] the degradation kinetics were studied by ultrasonication of solutions that contained palladium(II) dichloride bis(1dodecyl)diphenylphosphine, where the phosphine serves as a competing ligand. This favored chain scission under conditions that can be considered to provide kinetic control, whereas at long sonication times, the thermodynamic equilibrium is approached. An important factor limiting the selectivity is the position of the metal center in the original metallo-polymer. Degradation experiments with other polymers have established a minimum molecular weight of $c a .30^{\prime} 000 \mathrm{~g} \cdot \mathrm{mol}^{-1}$ for vinylic polymers such as poly(styrene) or poly(methyl methacrylate).[99] The macromonomers used in this study have a number-average molecular weight of $12000 \mathrm{~g} \cdot \mathrm{mol}^{-1}$ and therefore the relative position to the middle of the chain (where the mechanical force reaches its maximum) might significantly influence the selectivity due to a differing effective force.

Another metallopolymer that enabled the activation of latent catalytic species via ultrasonication was introduced by Bielawski and coworkers. $[16,100]$ The material comprises a di-palladium moiety, which is complexed by two pyridine end-capped poly(methacrylate) macro-ligands (Figure 27a). Upon ultrasonication of solutions containing the metallopolymer, the Pd-pyridyl bonds can be cleaved, which was confirmed via the activity of both fragments produced by the mechanically induced dissociation, i.e., the Pd-moiety as catalyst for the carbon-carbon formation between benzyl cyanide and Ntosyl imine, and the pyridyl-fragment, facilitating the anionic polymerization of an electron-poor acrylate ( $\alpha$-trifluoromethyl-2,2,2-trifluoroethyl acrylate). Both reactions do not proceed upon simply mixing or heating of the Pd-pyridyl metallo-polymer in the presence of the reactants. The authors clearly demonstrated that ultrasound-based mechanical activation of the latent polymeric catalyst is required. By variation of the molecular weight of the attached PMA tails, the authors also demonstrated that a limit molecular weight of $\sim 40^{\prime} 000 \mathrm{~g} \cdot \mathrm{mol}^{-1}$ was required to achieve efficient sonochemical activation.

a

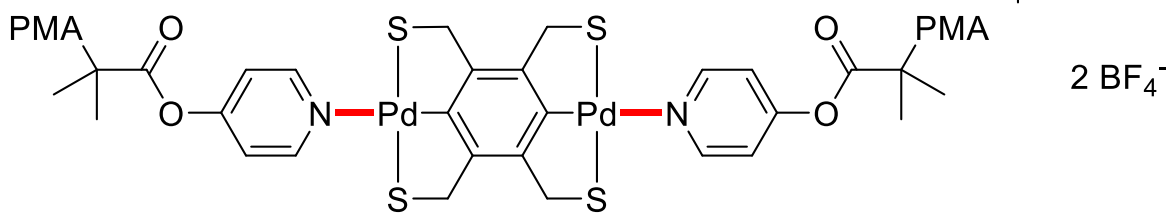

b<smiles></smiles>

Figure 27. Examples of mechanoresponsive latent polymer catalysts studied by Bielawski and coworkers. a) Polymer based on a pincer-type Pd-pyridiyl complex and poly(methacrylate) "tails". The Pd-pyridyl bonds highlighted in red can be cleaved by ultrasonication, revealing the Pd centers, which catalyze $\mathrm{C}-\mathrm{C}$ bond formation between benzyl cyanide and $\mathrm{N}$-tosyl imines. 
The corresponding pyridine-type ligand moiety can facilitate the polymerization of an electron-poor acrylate. b) Corresponding polymer comprising a mechanically responsive pyridine-[bis(pentafluorophenyl)]boron complex.

Bielawski and coworkers modified the above system by exchanging the Pd-pyridine complex against a boronium complex (Figure 27b).[101] The pyridine-boron bond is also sufficiently weak to permit sonochemical cleavage, enabling the pyridine moieties to initiate the anionic polymerization of $\alpha$-tri-fluoromethyl-2,2,2-trifluoroethyl acrylate. The cleavage of the metal-pyridine complex was colorimetrically confirmed by deprotonation of an indicator dye (4-[(4-anilinophenyl)azo]benzenesulfonic acid), whose color changed from deep purple to yellow, and by comparison with a reference polymer based on shorter PMA tails, which do not allow for a mechanical activation via ultrasound, since they are below the minimum threshold of length necessary to transduce the force from the cavitation bubbles. Another metallo-supramolecular system, on the basis of 2,6-bis(1,2,3triazol-4-yl)pyridine (BTP) as well as $\mathrm{Zn}^{2+}$ and $\mathrm{Eu}^{3+}$, respectively, was introduced by Weng and coworkers.[102] The approach is quite different from the majority of mechanically responsive metallo-supramolecular polymers heretofore described: the ligands were designed to be part of the polymer backbone instead of being located at the end of the polymer chains. Telechelic poly(tetrahydrofuran) (pTHF) was thus copolymerized with the di-hydroxy functionalized BTP ligand and hexamethylene diisocyanate to produce a polyurethane that was further cross-linked through the addition of metal ions (Figure 28a). The metal-rich domains phase-separate from the pTHF soft segments, forming a hard phase. This phase-segregated morphology results in additional physical crosslinks (Figure 28b). The material featured - depending on the ratio of BTP ligands to $\mathrm{Zn}^{2+}$ to $\mathrm{Eu}^{3+}$ ions - a high tensile strength (up to $18 \mathrm{MPa}$ ), a large strain at break (in excess of $1000 \%$, Figure $28 \mathrm{c}$ ) and a high toughness (up to $70 \mathrm{MPa}$ ). The metal-ligand hard phase was found to be thermally stable but mechanically labile. Mechanical stress caused the disruption of the hard phase domains and the dissociation of metal-ligand complexes, leading to a mechanical behavior that is reminiscent of the titin-mimics designed by the Guan group (vide supra). It is noted that in this case the mechanical properties are not the result of the unfolding of modular domains, but the metal-ligand based crosslinks appear to act like sacrificial bonds. The authors also demonstrated UV-light induced "reshuffling" of the supramolecular network, allowing for stress reduction upon irradiation with UV light on account of an increased dissociation rate of the metal-ligand complexes under these conditions. 
a

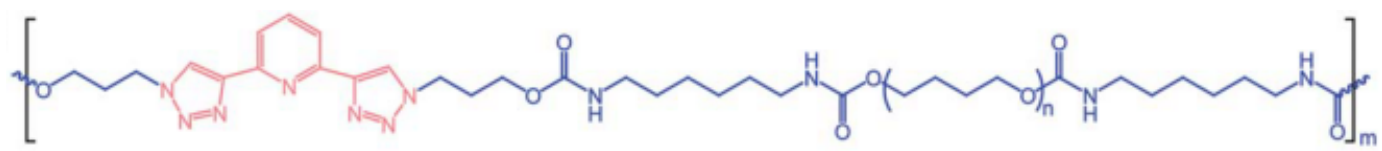

$\|$

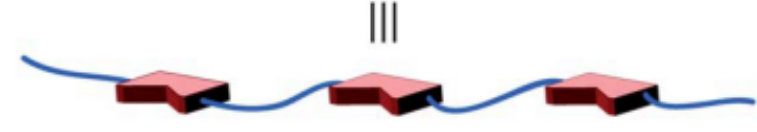

b

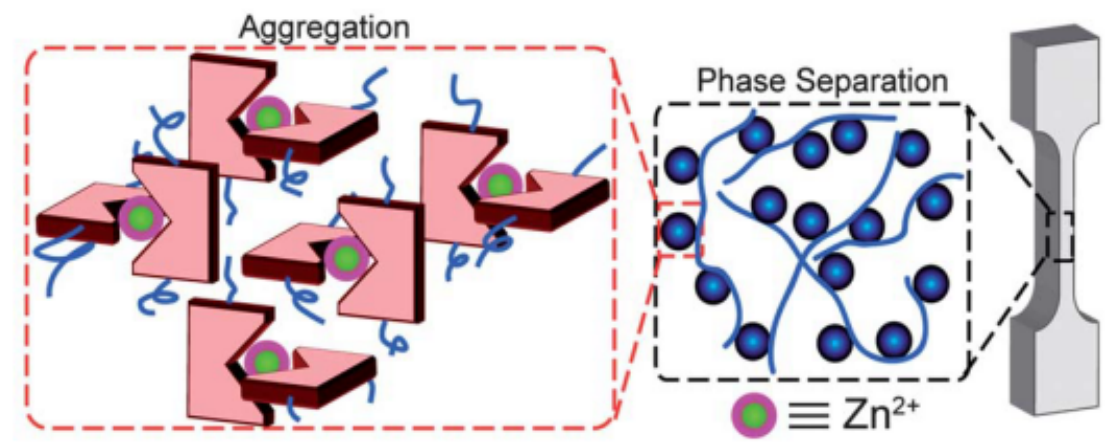

C

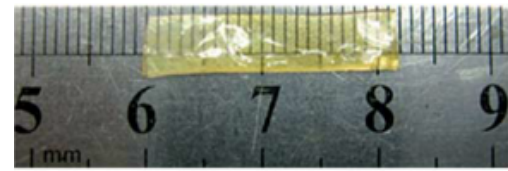

Figure 28. a) Chemical structure of a poly(THF)-BTP polyurethane. b) Schematic representation of structure and morphology of the metallo-supramolecular polymer network formed by combination of poly(THF)-BTP and $\mathrm{Zn}^{2+}$. c) Pictures of a film of the metallo-supramolecular polymer network made from poly(THF)-BTP and $\mathrm{Eu}^{3+}$ before and after stretching.[102] Adapted with permission from ref. [102]. Copyright 2013 The Royal Society of Chemistry.

Most recently, Liang et al. reported an example system for mechanically triggered drug release from metallo-supramolecular diblockcopolymer micelles (Figure 29).[103] The latter were based on bis(TPy)-Cu ${ }^{2+}$ (TPy $=2,2^{\prime}: 6^{\prime}, 2^{\prime}$ '-terpyridine) complexes that were employed to link poly(propylene glycol) (PPG) and poly(ethylene glycol) (PEG) into an amphiphilic blockcopolymer. The material was shown to able to self-assemble in aqueous solution into spherical micelles with hydrophobic PPG cores. The micelles can be opened/disrupted via ultrasound, which reversibly breaks the $\mathrm{Cu}^{2+}$-ligand complexes, and thereby allows the release of hydrophobic cargo molecules encapsulated in the micelles. After halting the sonication, the metal-ligand bonds were shown to re-form, resulting in the re-assembly of (slightly smaller) micelles. In reference experiments with micelle systems based on otherwise the same chemistry but stronger ruthenium-TPy complexes or covalent bonds (blockcopolymer PEO-PPG-PEO), no drug release under similar ultrasonication conditions was observed. 


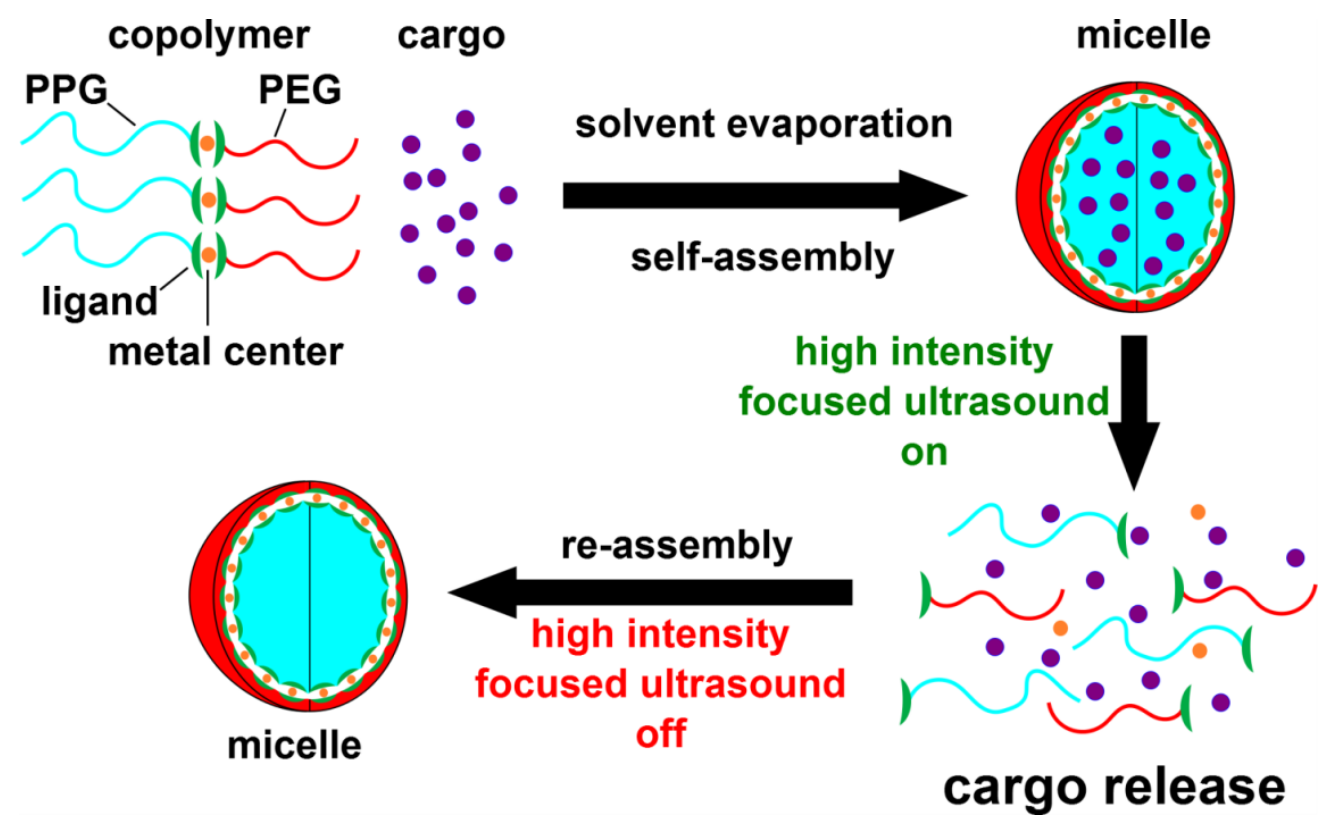

Figure 29. Schematic illustration of the self-assembly of amphiphilic metallo-supramolecular block-copolymers under encapsulation of cargo into micelles and disassembly of the micelles under release of the drug upon ultrasound sonication via dissociation of the metal-ligand complex. Upon cessation of the ultrasonication, the micelles re-assemble again.[103]

\section{Summary and Outlook}

Chemical bond activation through mechanical force, a hallmark of many biological materials, has long been known to occur in artificial materials, but such processes have traditionally been regarded as a challenge and not an opportunity. While mechanical force are well known to be capable of altering the electronic states of chemical bonds and thereby cause changes in chemical reactivity, optical properties, electrical conductivity, magnetic response, and other properties, it has been a veritable challenge to harness this knowledge to rationally design functional mechanoresponsive materials. The last decade, though, has witnessed tremendous progress in this domain. Researchers have been able to create mechanoresponsive systems in different materials families, including small organic molecules, polymers, and inorganic materials. These materials have exploited a broad range of mechanophores. While the development of the field of mechanically responsive polymers appears to have been catalyzed by early research involving supramolecular motifs, efforts have shifted in the last five years towards polymer comprising mechanophores that involve breaking of covalent bonds. Nevertheless, as summarized in this overview, a variety of supramolecular polymers have emerged in which exposure to mechanical force fields causes the selective dissociation of supramolecular motifs. Studies have evolved from proof-of-concept experiments to the design of a range of functional materials in which the mechanically triggered disassembly permits execution of a pre-programmed function. It appears that the general concept of mechanochemistry in supramolecular materials is versatile and can be exploited using a broad range of non-covalent interactions, that is, mechanophores that rely on hydrogen bonding, $\pi-\pi$ stacking, metal-ligand binding and other non-covalent bonds. As the strength of such interactions can be tailored over a broad range, and the molecular-level changes imparted by the (dis)assembly appear to be virtually limitless, his framework opens up avenues for the development of a plethora of new materials that are capable of transducing (weak) mechanical forces into a useful outcome, including, as discussed here, healing, color changes, calalytic function, and many others.

\section{Acknowledgments}

This work was supported by the National Center of Competence in Research (NCCR) Bio-Inspired Materials, a research instrument of the Swiss National Science Foundation. The authors acknowledge 
further support from the U.S. Army Research Office (W911NF-12-1-0339), the Swiss National Science Foundation (Grant No. 135405 and 152968), the European Research Council (ERC-2011AdG 291490-MERESPO), and the Adolphe Merkle Foundation. Y.S. acknowledges JSPS Postdoctoral Fellowships for Research Abroad.

\section{References}

1. Lumpkin EA, Caterina MJ (2007) Nature 445: 858. doi: 10.1038/Nature05662

2. Chen Y, Spiering AJH, Karthikeyan S, Peters GWM, Meijer EW, Sijbesma RP (2012) Nature Chemistry 4: 559. doi:10.1038/nchem.1358

3. Davis DA, Hamilton A, Yang JL, Cremar LD, Van Gough D, Potisek SL, Ong MT, Braun PV, Martinez TJ, White SR, Moore JS, Sottos NR (2009) Nature 459: 68. doi: 10.1038/Nature07970

4. Groote R, Jakobs RTM, Sijbesma RP (2013) Polymer Chemistry 4: 4846. doi: 10.1039/C3py00071k

5. Heinrichs A (2009) Nature Reviews Molecular Cell Biology 10: 163. doi: 10.1038/Nrm2642

6. Gillespie PG, Walker RG (2001) Nature 413: 194. doi: 10.1038/35093011

7. Brantley JN, Konda SSM, Makarov DE, Bielawski CW (2012) Journal of the American Chemical Society 134: 9882. doi: 10.1021/Ja303147a

8. Wiggins KM, Syrett JA, Haddleton DM, Bielawski CW (2011) Journal of the American Chemical Society 133: 7180. doi: 10.1021/Ja201135y

9. Lenhardt JM, Black AL, Craig SL (2009) Journal of the American Chemical Society 131: 10818. doi:10.1021/ja9036548

10. Beyer MK, Clausen-Schaumann H (2005) Chemical Reviews 105: 2921. doi: 10.1021/Cr030697h

11. Cravotto G, Cintas P (2012) Chemical Science 3: 295. doi: $10.1039 / \mathrm{C} 1 \mathrm{sc00740h}$

12. Chen YL, Spiering AJH, Karthikeyan S, Peters GWM, Meijer EW, Sijbesma RP (2012) Nature Chemistry 4: 559. doi: $10.1038 /$ Nchem. 1358

13. Lee CK, Davis DA, White SR, Moore JS, Sottos NR, Braun PV (2010) Journal of the American Chemical Society 132: 16107. doi: 10.1021/Ja106332g

14. Stuart MAC, Huck WTS, Genzer J, Muller M, Ober C, Stamm M, Sukhorukov GB, Szleifer I, Tsukruk VV, Urban M, Winnik F, Zauscher S, Luzinov I, Minko S (2010) Nature Materials 9: 101. doi: 10.1038/Nmat2614

15. Caruso MM, Davis DA, Shen Q, Odom SA, Sottos NR, White SR, Moore JS (2009) Chem. Rev. 109: 5755

16. Wiggins KM, Brantley JN, Bielawski CW (2012) Acs Macro Letters 1: 623. doi: 10.1021/Mz300167y

17. Black AL, Lenhardt JM, Craig SL (2011) Journal of Materials Chemistry 21: 1655. doi: 10.1039/C0jm02636k

18. Black AL, Orlicki JA, Craig SL (2011) Journal of Materials Chemistry 21: 8460. doi: 10.1039/C0jm03875j

19. Weder C (2015) In: Kobayashi S, Müllen K (eds) Encyclopedia of Polymeric Nanomaterials. Springer

20.

21.

22.

23.

24.

25. Weber CD, Robinson SG, Stay DP, Vonnegut CL, Lonergan MC (2012) ACS Macro Letters 1: 499.

Turro NJ (1978) Modern Molecular Photochemistry. University Science Books

Valeur B (2002) Molecular Fluorescence: Principles and Applications. Wiley-VCH

Birks JB (1975) Rep. Prog. phys. 38: 903.

Winnik FM (1993) Chemical Reviews 93: 587.

Goetz KP, Vermeulen D, Payne ME, Kloc C, McNeil LE, Jurchescu OD (2014) Journal of Materials Chemistry C 2: 3065. doi:10.1039/C3TC32062F doi:10.1021/mz300046x

26. M. Kasha, H. R. Rawls, El-Bayoumi MA (1965) Pure Appl. Chem. 11: 371.

27. Hong Y, Lamab JWY, Tang BZ (2011) Chem. Soc. Rev. 40: 5361.

28. Sagara Y, Kato T (2009) Nature Chemistry 1: 605. doi:10.1038/nchem.411

29. Pucci A, Ruggeri G (2011) Journal of Materials Chemistry 21: 8282. doi:10.1039/c0jm03653f

30. Pucci A, Bizzarri R, Ruggeri G (2011) Soft Matter 7: 3689. doi:10.1039/c0sm01038c

31. Ciardelli F, Ruggeri G, Pucci A (2013) Chem Soc Rev 42: 857. doi:10.1039/c2cs35414d

32. Makowski B, Kunzelman J, Weder C (2011) In: Urban M (ed) Handbook of Stimuli-Responsive materials. WileyVCH, New York p 117

33. Förster T, Kasper KZ (1954) Physik. Chem. NF 1: 275.

34. Förster T, Kasper KZ (1955) Elektrochem. Angew. Physik. Chem. 59: 976.

35. Döller E, Förster TZ (1962) Phys. Chem. 34: 132.

36. Birks JB, Dyson DJ, Munro IH (1963) Prog. Roy. Soc. 275 (Ser. A): 575.

37. Spies C, Gehrke RJ (2002) Phys. Chem. 106: 5348.

38.

39.

40.

Trabesinger W, A. Renn, B. Hecht, U. P. Wild, A. Montali, P. Smith, Weder C (2000) J. Phys. Chem. B $104: 5221$. Crenshaw BR, Weder C (2003) Chemistry of Materials 15: 4717. doi: 10.1021/Cm034447t

2400. doi:10.1021/ma062936j

41. Kinami M, Crenshaw BR, Weder C (2006) Chemistry of Materials 18: 946. doi:10.1021/cm052186c

42. Lott J, Weder C (2010) Macromolecular Chemistry and Physics 211: 28. doi:10.1002/macp.200900476

43. Crenshaw BR, Weder C (2006) Macromolecules 39: 9581. doi:10.1021/ma061685b

44. Kunzelman J, Crenshaw BR, Kinami M, Weder C (2006) Macromolecular Rapid Communications 27 : 1981. doi:10.1002/marc.200600642

45. Kunzelman J, Gupta M, Crenshaw BR, Schiraldi DA, Weder C (2009) Macro. Mater. Eng. $294: 244$. doi:10.1002/mame.200800299

46. Crenshaw BR, Weder C (2005) Advanced Materials 17: 1471. doi: 10.1002/adma.200401688 
47. Crenshaw BR, Kunzelman J, Sing CE, Ander C, Weder C (2007) Macromolecular Chemistry and Physics 208: 572. doi:10.1002/macp.200600622

48. Kunzelman J, Chung T, Mather PT, Weder C (2008) Journal of Materials Chemistry 18: 1082. doi:10.1039/b718445j

49. Sing CE, Kunzelman J, Weder C (2009) Journal of Materials Chemistry 19: 104. doi:10.1039/b813644k

50. Kunzelman J, Crenshaw BR, Weder C (2007) Journal of Materials Chemistry 17: 2989. doi:10.1039/b705880b

51. Tang L, Whalen J, Schutte G, Weder C (2009) ACS Appl Mater Interfaces 1: 688. doi:10.1021/am800199u

52. $\quad$ Pucci A, Bertoldo M, Bronco S (2005) Macromolecular Rapid Communications 26: 1043. doi:10.1002/marc.200500227

53. Pucci A, Di Cuia F, Signori F, Ruggeri G (2007) Journal of Materials Chemistry 17: 783. doi:10.1039/b612033d

54. Donati F, Pucci A, Cappelli C, Mennucci B, Ruggeri G (2008) J. Phys. Chem. B 112: 3668.

55. Lowe C, Weder C (2002) Advanced Materials 14: 1625. doi: 10.1002/1521-4095(20021118)14:22<1625::AidAdma1625>3.0.Co;2-Q

56. Löwe C, Weder C (2002) Synthesis 9: 1185.

57. Makowski BT, Lott J, Valle B, Singer KD, Weder C (2012) Journal of Materials Chemistry 22: 5190. doi:10.1039/c2jm15846a

58. Kunzelman J, Kinami M, Crenshaw BR, Protasiewicz JD, Weder C (2008) Advanced Materials 20: 119. doi:10.1002/adma.200701.772

59. Sagara Y, Komatsu T, Ueno T, Hanaoka K, Kato T, Nagano T (2014) J Am Chem Soc 136: 4273. doi:10.1021/ja412670g

60. Burattini S, Colquhoun HM, Greenland BW, Hayes W (2009) Faraday Discuss. 143: 251.

61. Burattini S, Colquhoun HM, Fox JD, Friedmann D, Greenland BW, Harris PJ, Hayes W, Mackay ME, Rowan SJ (2009) Chem Commun (Camb): 6717. doi:10.1039/b910648k

62. Burattini S, Greenland BW, Merino DH, Weng W, Seppala J, Colquhoun HM, Hayes W, Mackay ME, Hamley IW, Rowan SJ (2010) J. Am. Chem. Soc. 132: 12051.

63. Hart LR, Hunter JH, Nguyen NA, Harries JL, Greenland BW, Mackay ME, Colquhoun HM, Hayes W (2014) Polymer Chemistry 5: 3680. doi:10.1039/c4py00292j

64. Brunsveld L, Folmer BJB, Meijer EW, Sijbesma RP (2001) Chemical Reviews 101: 4071. doi:10.1021/cr990125q

65. Hoeben FJM, Jonkheijm P, Meijer EW, Schenning A (2005) Chemical Reviews 105: 1491. doi:10.1021/cr030070z

66. Aida T, Meijer EW, Stupp SI (2012) Science 335: 813. doi:10.1126/science. 1205962

67. Kato T, Mizoshita N, Kishimoto K (2005) Angew Chem Int Ed Engl 45: 38. doi:10.1002/anie.200501384

68. Wojtecki RJ, Meador MA, Rowan SJ (2011) Nature Materials 10: 14. doi:10.1038/nmat2891

69. Guimard NK, Oehlenschlaeger KK, Zhou J, Hilf S, Schmidt FG, Barner-Kowollik C (2012) Macromolecular Chemistry and Physics 213: 131. doi:10.1002/macp.201100442

70. Sijbesma RP, Beijer FH, Brunsveld L, Folmer BJB, Hirschberg JHKK, Lange RFM, Lowe JKL, Meijer EW (1997) Science 278: 1601.

71. Sivakova S, Bohnsack DA, Mackay ME, Suwanmala P, Rowan SJ ( 2005) J. Am. Chem. Soc. 127: 18202.

72. Guan Z, Roland JT, Bai JZ, Ma SX, McIntire TM, Nguyen M (2004) J. Am. Chem. Soc. 126: 2058.

73. Roland JT, Guan Z (2004) J. Am. Chem. Soc. 126: 14328.

74. Kushner AM, Gabuchian V, Johnson EG, Guan Z (2007) J. Am. Chem. Soc. 129: 14110.

75. Kushner AM, Vossler JD, Williams GA, Guan Z (2009) J. Am. Chem. Soc. 131: 8766.

76. Beck JB, Rowan SJ (2003) Journal of the American Chemical Society 125: 13922. doi:10.1021/ja038521k

77. Lis S (2002) Journal of Alloys and Compounds 341: 45. doi:http://dx.doi.org/10.1016/S0925-8388(02)00055-5

78. $\quad$ Sabbatini N, Guardigli M, Lehn J-M (1993) Coordination Chemistry Reviews 123: 201. doi:http://dx.doi.org/10.1016/0010-8545(93)85056-A

79. Schubert US, Eschbaumer C (2002) Angewandte Chemie International Edition 41: 2892. doi:10.1002/15213773(20020816)41:16<2892::AID-ANIE2892>3.0.CO;2-6

80. Rowan SJ, Beck JB (2005) Faraday Discussions 128: 43. doi:10.1039/B403135K

81. Kumpfer JR, Wie JJ, Swanson JP, Beyer FL, Mackay ME, Rowan SJ (2011) Macromolecules 45: 473. doi:10.1021/ma201659d

82. Burnworth M, Tang L, Kumpfer JR, Duncan AJ, Beyer FL, Fiore GL, Rowan SJ, Weder C (2011) Nature 472: 334. doi:10.1038/nature09963

83. Coulibaly S, Roulin A, Balog S, Biyani M, Foster EJ, Rowan SJ, Fiore GL, Weder C (2014) Macromolecules 47: 152.

84. Biyani MV, Foster EJ, Weder C (2013) ACS Macro Letters 2: 236-240.

85. Fiore G, Rowan SJ, Weder C (2013) Chem. Soc. Rev. 42: 7278.

86. Balkenende DWR, Coulibaly S, Balog S, Simon YC, Fiore GL, Weder C (2014) Journal of the American Chemical Society 136: 10493. doi: $10.1021 / \mathrm{Ja} 5051633$

87. Simon YC, Fiore GL, Weder C (2014) Chimia 68: 666. doi: 10.2533/chimia.2014.666

88. Caruso MM, Davis DA, Shen Q, Odom SA, Sottos NR, White SR, Moore JS (2009) Chemical Reviews 109: 5755. doi: $10.1021 / \mathrm{Cr} 9001353$

89. Kumpfer JR, Taylor SD, Connick WB, Rowan SJ (2012) Journal of Materials Chemistry 22: 14196. doi:10.1039/C2JM32160B

90. Paulusse JMJ, Sijbesma RP (2004) Angewandte Chemie International Edition 43: 4460. doi:10.1002/anie.200460040

91. Groote R, Szyja BM, Leibfarth FA, Hawker CJ, Doltsinis NL, Sijbesma RP (2014) Macromolecules 47: 1187. doi:10.1021/ma4022339 
92. Jakobs RTM, Ma S, Sijbesma RP (2013) ACS Macro Letters 2: 613. doi:10.1021/mz400201c

93. Groote R, Jakobs RTM, Sijbesma RP (2013) Polymer Chemistry 4: 4846. doi:10.1039/c3py00071k

94. Jakobs RTM, Sijbesma RP (2012) Organometallics 31: 2476. doi: 10.1021/Om300161z

95. Paulusse JMJ, Huijbers JPJ, Sijbesma RP (2006) Chemistry - A European Journal 12: 4928. doi:10.1002/chem.200600120

96. Paulusse JMJ, van Beek DJM, Sijbesma RP (2007) Journal of the American Chemical Society 129: 2392. doi:10.1021/ja067523c

97. Paulusse JMJ, Sijbesma RP (2008) Chemical Communications: 4416. doi:10.1039/B806978F

98. Berkowski KL, Potisek SL, Hickenboth CR, Moore JS (2005) Macromolecules 38: 8975. doi:10.1021/ma051394n

99. Nguyen TQ, Liang OZ, Kausch HH (1997) Polymer 38: 3783. doi: 10.1016/S0032-3861(96)00950-0

100. Tennyson AG, Wiggins KM, Bielawski CW (2010) Journal of the American Chemical Society 132: 16631. doi:10.1021/ja107620y

101. Wiggins KM, Hudnall TW, Tennyson AG, Bielawski CW (2011) Journal of Materials Chemistry 21: 8355. doi:10.1039/C0JM03619F

102. Yuan J, Zhang H, Hong G, Chen Y, Chen G, Xu Y, Weng W (2013) Journal of Materials Chemistry B 1: 4809. doi:10.1039/C3TB20647E

103. Liang B, Tong R, Wang Z, Guo S, Xia H (2014) Langmuir 30: 9524. doi:10.1021/la500841x 